\title{
Applying Artificial Intelligence Techniques for Prediction of Neurodegenerative Disorders: A Comparative Case-Study on Clinical Tests and Neuroimaging Tests with Alzheimer's Disease
}

\author{
Ahmed Abdullah Farid ${ }^{1}$, Gamal Ibrahim Selim¹, Hatem Awad A. Khater ${ }^{\mathbf{3}}$ \\ ${ }^{1}$ Department of Computer Engineering, Faculty of Engineering \& Technology, Arab Academy for Science \\ Technology and Maritime Transport(AASTMT), Cairo, Egypt \\ ${ }^{3}$ Associate Professor Dr. Hatem Awad Aly Khater, Faculty of Engineering, Horus University in Egypt
}

\begin{abstract}
Alzheimer's disease (AD) detection acting as an essential role in global health care due to misdiagnosis and sharing many clinical sets with other types of dementia, and costly monitoring the progression of the disease over time by magnetic reasoning imaging (MRI) with consideration of human error in manual reading. This paper goal a comparative study on the performance of data mining techniques on two datasets of Clinical and Neuroimaging Tests with AD. Our proposed model in the first stage, apply clinical medical dataset to a composite hybrid feature selection (CHFS), for extract new features to select the best features due to eliminating obscures features, In parallel with Apply a novel hybrid feature extraction of three batch edge detection algorithm and texture from MRI images dataset and optimized with fuzzy 64-bin histogram. In the second stage, we applied a clinical dataset to a stacked hybrid classification(SHC) model to combine Jrip and random forest classifiers with six model evaluations as meta-classifier individually to improve the prediction of clinical diagnosis. At the same stage of improving the classification accuracy of neuroimaging (MRI) dataset images by applying a convolution neural network (CNN) in comparison with traditional classifiers, running on extracted features from images. The authors have collected the clinical dataset of 426 subjects with (1229 potential patient sample) from oasis.org and (MRI) dataset from a benchmark kaggle.com with a total of around $~ 5000$ images each segregated into the severity of Alzheimer's. The datasets evaluated using an explorer set of weka data mining software for the analysis purpose. The experimental show that the proposed model of (CHFS) feature extraction lead to effectively reduced the falsenegative rate with a relatively high overall accuracy with a stack hybrid classification of support vector machine (SVM) as meta-classifier of $96.50 \%$ compared to $68.83 \%$ of the previous result on a clinical dataset, Besides a compared model of CNN classification on MRI images dataset of $80.21 \%$. The results showed the superiority of our CHFS model in predicting Alzheimer's disease more accurately with the clinical medical dataset in early-stage compared with the neuroimaging (MRI) dataset. The results of the proposed model were able to predict with accurately classify Alzheimer's clinical samples at a low cost in comparison
\end{abstract}


with the MRI-CNN images model at the early stage and get a good indicator for high classification rate for MRI images when applying our proposed model of SHC.

Keywords: Data Mining, Alzheimer's Dementia, Composite Hybrid Feature Selection, Machine learning, stack Hybrid Classification, AI, MRI, Neuroimaging, MPEG7 edge histogram feature extraction, $\mathrm{CNN}$.

\section{Introduction}

Data mining skills involved in biomedical sciences and investigate for providing prediction for help to identify the disease and classify it correctly (Kalló, Gergő \& Miklós Emri et al., 2016) (K. Tejeswinee \& S.G. Jacob.,2017). (J. Escuderoaccess et al., 2013) (Chi, C. L. \& Oh, W. \& Borson \& S.,2015). AD is a form of dementia that shows for $60-80 \%$ of mental disorders (David P Salmon \& Mark W. Bondi,2010). AD is the sixth cause of death in the united states, according to the national center for health statistics 2019 (CDC) (the online NCHR Report,2019). Clinical AD research can create a new challenge for the possibility of effective treatment (Williams \& Jennifer A.,2013) (Klöppel \& ...\&Frackowiak \& R. Set al.,2008). There are two methods of medical diagnosis for AD, Neuroimaging MRI \& PET scans, and clinical tests of (CDR \& MMSE \& Memory) (Dai \& C. Lu,2013). Our proposed model using composite hybrid attribute selection (CHFS) in the clinical dataset to achieve high accuracy in prediction and improve the feature extraction methods (Chen\&..\& Lin,2005)(Dai \& C. Lu,2013) with hybrid classification techniques for combine multiclassifiers to improving an in-depth investigation. In addition to the investigation of the Kaggle medical dataset with 5000 MRI images used to train and test mode in a comparative study between the neuroimaging dataset and clinical test in early-stage and which one can provide us the heigh accurate prediction in a low cost. In the MRI dataset, Our proposed hybrid feature extraction of four filters ( MPEG-7 edge histogram filter with Gabor filterpyramid of rotation-invariant local binary pattern histograms - fuzzy 64-bin histogram )(A. Roberts,2012)(G.Saianilkumar,2015) which analyzes a low-level feature of an image can extract the features and provide a statistical hypothesis. Testing is employed to see which feature vectors/elements are most informative to differentiate different image classes. Also, using $(\mathrm{CNN})$ for relatively little pre-processing compared to other image classification algorithms and traditional classifiers. The article planned as follows. The next section discusses the literature review of other authors who have used data mining and its relative of machine learning algorithm to analyze and diagnose Alzheimer's disease and various diseases by clinical tests and neuroimaging of MRI and PET scans. Section 3 describes the proposed technique used for feature extraction in two medical datasets with the CHFS model and four image filters for extract features from MRI images. Section 4 describes the method used for the hybrid classification process in the clinical dataset and convolution neural network (CNN) in comparison with traditional classifiers, whereas section 5 describes the experiments and evaluation. Section 6 discusses the results. Finally, section 7 presents the paper summary and conclusions. 


\section{Literature Review}

Many researchers have used data mining for the diagnosis of various diseases. Some of them are Jyothi Sony has used classifiers, namely naïve Bayes, k-Nearest Neighbors. Decision tree, to predict heart disease (Jyoti Soni.,2010)(Williams \& Jennifer A.,2013) record clinical dementia rating (CDR)by support vector machine (SVM) and decision tree with a neural network and naive Bayes replaced missing value with average one to achieve best accuracy and correlation (Chi, C. L. \& Oh, W. \& Borson \& S.,2015). Voxel-based morphometry applied to MRI images from an oasis medical dataset (Chyzhyk \& Savio,2010) (online access Oasis Medical Dataset,2019) . S. R. Bhagya Shree et al. compared many classifiers such as naïve Bayes, decision tree algorithm J48, random forest, JRip in the detection of Alzheimer's disease (S. R Bhagya Shree \& Dr. H. S. Sheshadri,2014), the results indicated naïve Bayes, Jrip and random forest perform better, the problem with this paper was the data set was having records of 250 subjects and the data not preprocessed.

Tina R. Patil et al., in their paper 'performance analysis of ayes and J48 classification algorithm for data classification,' has discussed naive Bayes classification (Tina R. Patil \& Mrs. S. S. Sherekar,2013) . Jehad Ali et al., in their paper, has discussed Random forest and J48 for the classification of data sets (Jihad Ali et al. ,2012), The prediction of Alzheimer's disease using SVM on the MRI picture implemented by Vemuri, P., Gunter(Vemuri \& ...Jack \& C. R.,2008) .

In more recent developments, Casanova et al. (Ramon Casanova,2013) introduced new metrics for assessing Alzheimer's Disease risk based on Structural Magnetic Resonance Imaging (SMRI) and cognitive performance data. They refer to these metrics as Alzheimer's Disease Pattern Similarity (AD-PS) scores. Using data from the Alzheimer's Disease Neuroimaging Initiative (ADNI), they calculated conditional probabilities modeled by largescale regularized logistic regression. They conclude that AD-PS metrics could be a powerful tool in Alzheimer's Disease research to detect Alzheimer's Disease-like cognitive and anatomical effects and that this approach could extend to other diseases such as Parkinson's disease.

The Alzheimer's malady Neuroimaging activity, portrayed in(Susanne G Mueller,2005), started in 2004 and had the general goal of describing clinical, hereditary, imaging, and biochemical biomarkers of the sickness and recognizing the connections between them throughout malady movement from typical insight to MCI to dementia. It likewise settled vaults of information and organic examples, the two of which were to be uninhibitedly open to the more extensive scholarly and explore the network. Potential utilization of these archives as a reason for examinations utilizing $\mathrm{AI}$ is to use them as preparing sets for the making of classifiers, for example, SVMs (Bolster Vector Machines) or Choice trees.

(Elizabeth G Kehoe,2005) note that structural X-ray proportions of the hippocampus and average worldly flap are as yet, the most clinically approved biomarkers for Alzheimer's Infection, be that as it may. More current procedures, for example, practical X-ray and dispersion tensor imaging, another extraordinary scope in following changes in the mind, especially in useful and auxiliary availability, which may go before dim issue decay. That is a significant severe articulation furthermore, mirrors the present perspective on neuroimaging based conclusion of Advertisement. 
In 2015 the Informatics center at ADNI (W Toga and Karen L,2015) distributed an audit of the first decade of their information assortment and spread. In the survey, they report that ADNI scatters information to a consistently developing number of agents who have composed many scientific papers dependent on ADNI information. The ADNI itself (Michael Weiner,2015) says that roughly 500 articles have been distributed as an immediate aftereffect of ADNI as far as possible of 2013. Research utilizing ADNI information crosses numerous researcher disciplines, geographic areas. It incorporates PC researchers keen on creating and testing AI and characterization calculations, neuroscientists, interested in developing and researching disease development models, radiologists, geneticists, and many others looking to expand scientific knowledge limits.

Modern image edge detection algorithms include both first-order differential operators (i.e., operators Roberts, Prewitt, Sobel, and Canny) and second-order differential operators (i.e., operators Laplacian which LoG) and can extend to a wide variety of applications. Through integrating this with mathematical mechanics, Wang and Liu used the Roberts operator to identify vehicle image edges and distinguish vehicle license plate positions.

The following conclusions were drawn when analyzing findings with existing studies. While the detection mentioned above algorithms have the benefits of being simple and easy to implement and delivering excellent performance in real-time, they also have obvious shortcomings. The Roberts operator extracted image edge function is relatively rough and offers imprecise edge positions. The edge features which the Prewitt operator extracts have wide margins and many discontinuities. Likewise, the Sobel director does not provide precise picture edge coordinates. The Laplacian operator highly noised sensitive, and the LoG operator can not remove salt and pepper noise in an image.

\section{Proposed Work}

In the proposed work, the clinical medical dataset collected from the oasis brain organization (online access Oasis Medical Dataset,2019). The medical dataset contains several clinical tests of non-demented and demented older adults. The proposed layout in Fig 1.2

Fig. 1 The proposed layout for the clinical dataset

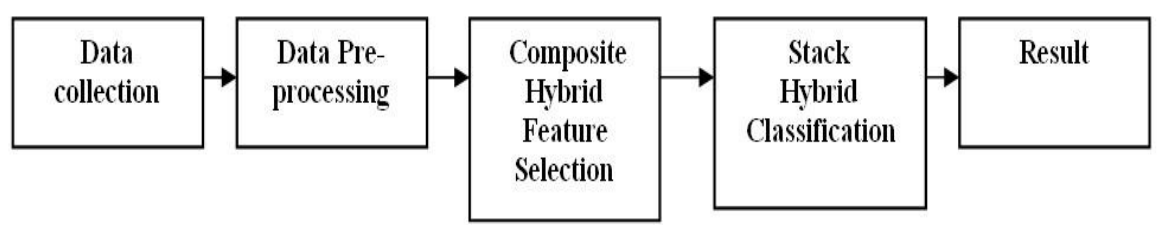


Fig. 2 The proposed design for the neuroimaging MRI dataset

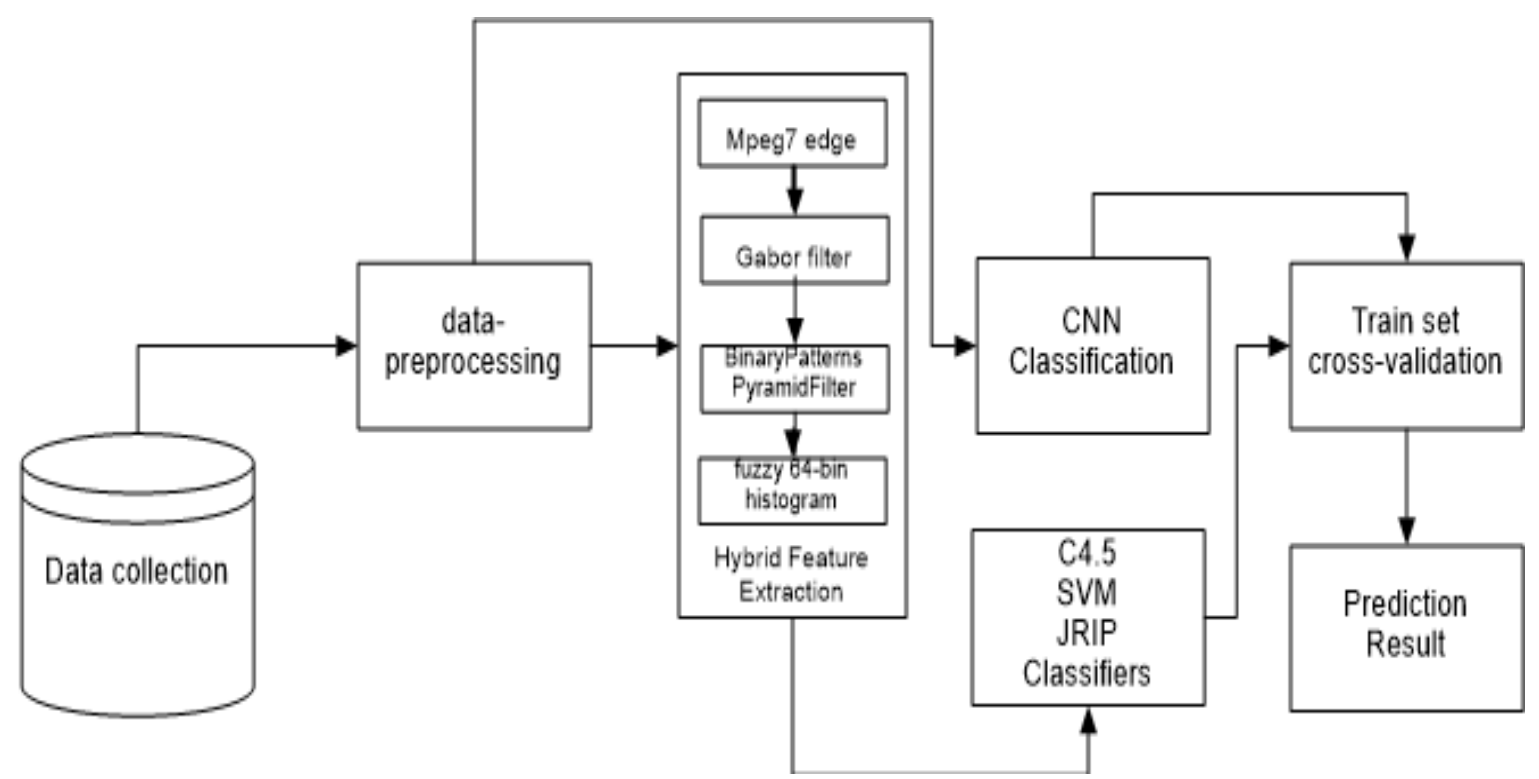

The following steps explain the mechanism of the proposed work in the clinical dataset and MRI dataset :

\subsection{Data Collection}

A. The clinical medical dataset contains 426 subjects with 1229 records of potential patients (online access Oasis Medical Dataset,2019) .

B. The images MRI segmentation AD dataset has four classes of images both in training as well as the testing set containing a total of around $\sim 5000$ images each segregated into the severity of Alzheimer's (online access Kaggle benchmark dataset,2019):
i. $\quad$ Mild Demented
ii. Moderate Demented
iii. Non Demented
iv. Very Mild Demented

\subsection{Data Pre-processing}

In the real world, data collected tend to be not wholly complete, noisy and conflicting, detection missing of data, data irregularity, prevent the errors, and decrease the data to be analyzed would lead to massive payouts for decision making (H. Witten \& Eibe Frank ,2008)

For machine learning and analytics, the selection of features is the process of selecting a subset of specific features (variables, predictors) for use in model building. Component selection methods used for many reasons(H. Witten \& Eibe Frank ,2008):

i. simplification of the models to make them easier for researchers/users to understand

ii. shorter training periods

iii. preventing the curse of dimensionality

iv. improved generalization by minimizing overfitting 


\subsection{A. Proposed Composite Hybrid Feature Selection Model (CHFS) to Clinical Dataset}

Feature selection is the approach of taking a subset of relevant features for use in model construction(Chen \& ...\& F. Li,2010), and combines the advantages of three feature selection approaches (Filter (IG,GR)-Wrapper( improved (Genetic Algorithm)) with Embedded(C4.5)).

\subsection{A.1 Composed Hybrid feature selection architecture}

The author combine of three feature extraction technique considered for the optimal selection feature set, and this method is information gain (IG) - gain ratio (GR) and Optimized Genetic Algorithm(Huang \& C.,2012) (Yanan Mao \& Dingyuan Fan,2016).as shown by fig 3

Fig.3 The proposed composite hybrid feature selection architecture

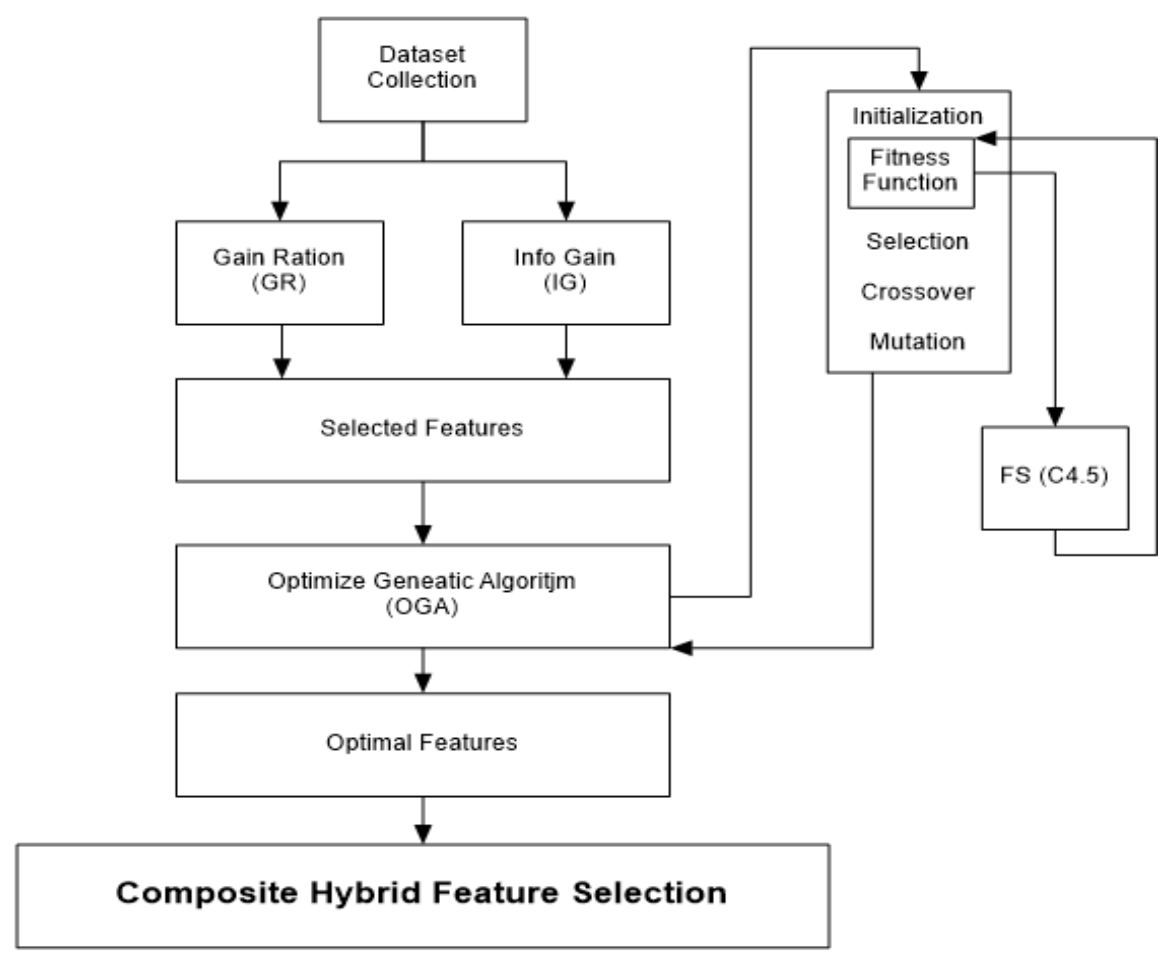

In Figure 2, The superior ranked features taken as the optimized subset, the threshold established the size of the resumption feature subset and amount of attributes in the dataset that appear in the range(7 10) (Vemuri \& et al.Jack \& C. R.,2008) (Devi \&Cuckoo,2013)...

\subsection{A.2 Information Gain feature selection}

The calculation of the information gain for only one attribute according to the algorithm below (Aouatif Amine \& ...\& Rziza Driss ,2011): This gain measure gives the effect of the features, and the following algorithm selects features that are larger than the threshold.

\subsection{A.3 Gain Ratio Feature Selection}

A decision tree can be a simple form when non-terminal nodes perform tests on many attributes to the effect of decision outcomes (J.R. Quinlan,1986). The gain ratio is

$$
\operatorname{GainRatio}(A)=\operatorname{Gain}(A) / \operatorname{QplitInfo} A(Q)
$$




\subsection{A.4 Optimized Genetic Algorithm (OGA)}

The authors propose a method to modify a general genetic algorithm to evaluates specified attributes on training data or a separate testing set and uses a decision tree (J.R. Quinlan,1986) to estimate the 'merit' of a set of attributes to produce an optimized feature subset with genetic search elevation strategy to recognize the features. All feature selection technique should use an evaluation function together with a search strategy to achieve the optimal feature set(Huang \& C.,2012).

It is unable to be realized to search all subsets to find out an optimal subset and need much effort to indicate whether a particular feature is present or not in the chromosome, one, and zero used. One in a gene position refers to feature and zero to absent(Yanan Mao \& Dingyuan Fan,2016). The number of features and what are the features that are to be present in a chromosome are guided by information gain (IG) and gain ratio (GR).

The initial population created using input values of IG and GR of the values present in the chromosome. After Generated the population, the individuals evaluated using a fitness function. There is no general approach to find the fitness function for a genetic algorithm. It is a heuristic approach and depends on the used application. So the authors nominate a C4.5 classifier to be used as a fitness function because $\mathrm{C} 4.5$ has some utility of handling both continuous and discrete attributes and training data with missing attribute values, pruning trees after creation - C4.5 goes back through the tree once it has been created and try to eject branches that do not help by replacing them with leaf nodes (Dash \& H. Liu, 1997) . (J.R. Quinlan,1986). The following algorithm selects a feature from the set of features that are gained by OGA, gain ratio, and Information gain, as shown in fig 4.

Fig. 4 Proposed optimization of a genetic algorithm with the c4.5 decision tree

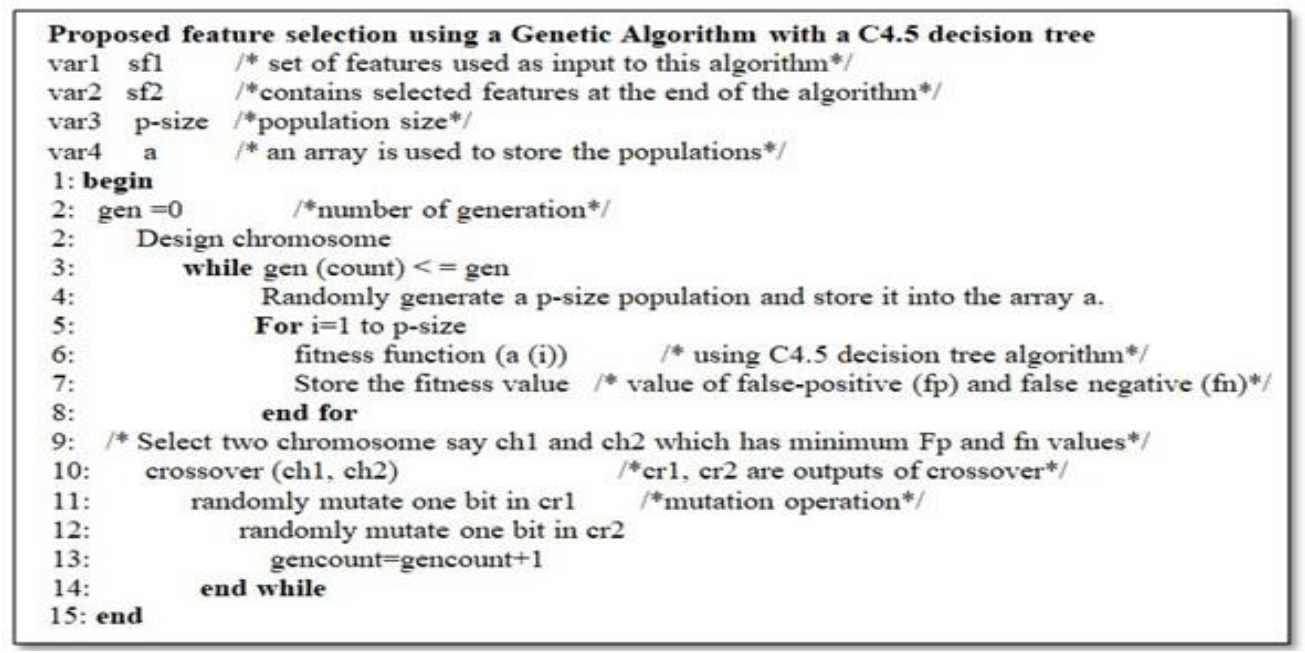




\subsection{B proposed Hybrid Feature Selection Approach on MRI Images}

There is a variability of approaches used to obtain images. Some of these are the mean and difference dependent on the bi-orthogonal wavelet filter, image retrieval based on shape. Edges are an essential image feature that is present between an objective and a background and between two targets, two zones, or two primitives. Much of the details for a picture borne in the margins. A picture edge is usually a group of pixels for which the gray-level values demonstrate a shift in phase (Jianfang Cao,2018).

\subsection{B.1 MPEG7 Histogram Filter}

The histogram is the form most widely used to describe an image's composition of any global function. Translation and rotation of the picture invariant, a normalization of the

$$
\begin{aligned}
& G_{c}[i, j]=B e^{-\frac{\left(i^{2}+j^{2}\right)}{2 \sigma^{2}}} \cos (2 \pi f(i \cos \theta+j \sin \theta)) \\
& G_{s}[i, j]=C e^{-\frac{\left(i^{2}+j^{2}\right)}{2 \sigma^{2}}} \sin (2 \pi f(i \cos \theta+j \sin \theta))
\end{aligned}
$$
histogram corresponds to the 7 , invariance of the distance.

According to the edge histogram description in MPEGan extra histogram bin may easily be created from the local 5-bin edge histogram of each $4 * 4$ sub-image. A statistical hypothesis testing is employed to see which feature vectors/elements are most informative to differentiate different image classes. The histogram is very useful for indexing and extracting pictures using the above property.

\subsection{B.2 Gabor Image Filter}

Gabor filter used for texture analysis, which implies that it mainly analyzes if there is some different frequency information in the picture in specific directions across the point or area of analysis in a regional region. It can define by a sinusoidal wave (a plane wave for 2D Gabor filters) multiplied by a Gaussian function.

A set of Gabor filters with different frequencies and orientations may assist in extracting useful features from an image. In the discrete domain, the two-dimensional Gabor filters are given by(2)-(3),

\subsection{B.3 Pyramid of Rotation-Invariant Local Binary Pattern Histograms Image Filter}

The local binary sequence (LBP) commonly used in the classification of textures. The modern LBP methods define only micro-texture picture structures, such as edges, corners, points, although many of them show excellent texture classification efficiency. This situation could still not be changed, although the technique of multi-resolution research used in local binary pattern methods(Ojala et al., 2002). The texture operator LBP has become a simplified 
approach in different applications. These can describe as a unifying solution to historically different computational and structural texture research frameworks.

To the LBP operator, the following terminology used: LBPP, Ru2. The subscript represents a neighborhood using the operator $(\mathrm{P}, \mathrm{R})$. Superscript $\mathrm{u} 2$ indicates that only standard patterns used and the other patterns marked with a single label. After obtaining the LBP-labeled picture $\mathrm{fl}(\mathrm{x}, \mathrm{y})$, the LBP histogram can identify as

$$
H i=\sum x, y I\{f l(x, y)=i\}, i=0, \ldots, n-1,
$$

$\mathrm{n}$ is the number of different LBP operators labels, and I\{A $\}$ is 1 if $\mathrm{A}$ is valid and 0 if $\mathrm{A}$ is false. When the picture patches with different sizes applied to the histograms, the histograms must standardized to achieve a coherent description:

$$
N i=H_{i} \sum n-1 j=0 H_{j}
$$

\subsection{B.4 Fuzzy 64-bin Histogram Image Filter}

The Fuzzy 64-bin focused on color vision is usually not typically shown in RGB. The better model of HVS is the so-called opposing color type. The competing color space has three component fuzzy 64-bin bases on color expectations typically not better represented in RGB. (C. A. Bouman,2007):

- $\mathrm{O} 1$ is a luminance component

- $\mathrm{O} 2$ is the red-green channel

- $\mathrm{O} 2=\mathrm{G}-\mathrm{R}$

- $\mathrm{O} 3$ is the blue-yellow channel

- $\mathrm{O} 3=\mathrm{B}-\mathrm{Y}=\mathrm{B}-(\mathrm{R}+\mathrm{G})$

Consequences of Opponent Channel CSF

Luminance channel is Bandpass function

- Wide bandwidth $\Rightarrow$ high spatial resolution.

- Low-frequency cut-off is $\Rightarrow$ insensitive to the average luminance level.

Chrominance channels are

- Lowpass function

- Lower bandwidth $\Rightarrow$ low spatial resolution.

- Low pass $\Rightarrow$ sensitive to absolute chromaticity (hue and saturation). ( C. A. Bouman, 2007)

\subsection{B.3 Applying Hybrid feature selection architecture model on MRI images}

The author combines four filters to feature extraction process considered for the optimal selection feature set from MRI images, as shown in fig 5. 


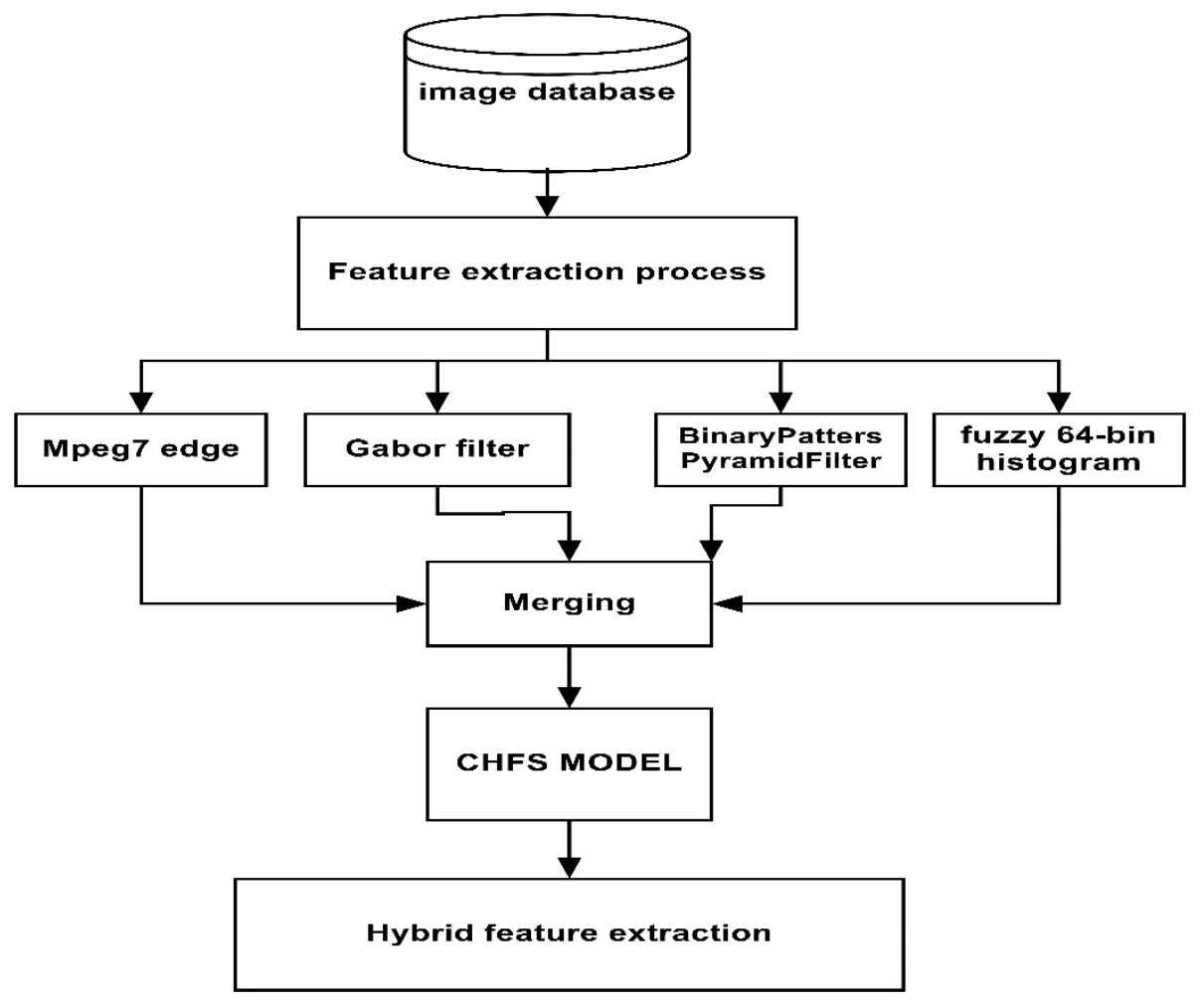

\section{Proposed Stack Hybrid Classification Model Based On Composite Hybrid Feature Selection (CHFS) on Clinical Dataset}

A weka software tool (Weka online open-source accessed,2018) shows the list of black-box classifiers. These algorithms, in general, are used to classify the medical dataset.

\subsection{A. Two learning evaluators can be used to evaluate the dataset}

Training set: the classifier separates a dataset to test and training data. And Cross-validation: in case of 10 fold cross-validation (Divya Jain, Vijendra Singh,2018).

\subsection{B. Stacking technique}

Ensemble methods are learning methods that contain a set of classifiers for classifying data by taking a weighted point of their predictions (Leo Breiman,1996). The authors combine multiple classifiers to get the maximum efficiency of classification accuracy and overcome the weakness of individual classifiers in the classification process on potential patients. Classifiers, as shown in fig 6. 
Fig. 6the proposed framework of stack hybrid classification based on the CHFS model

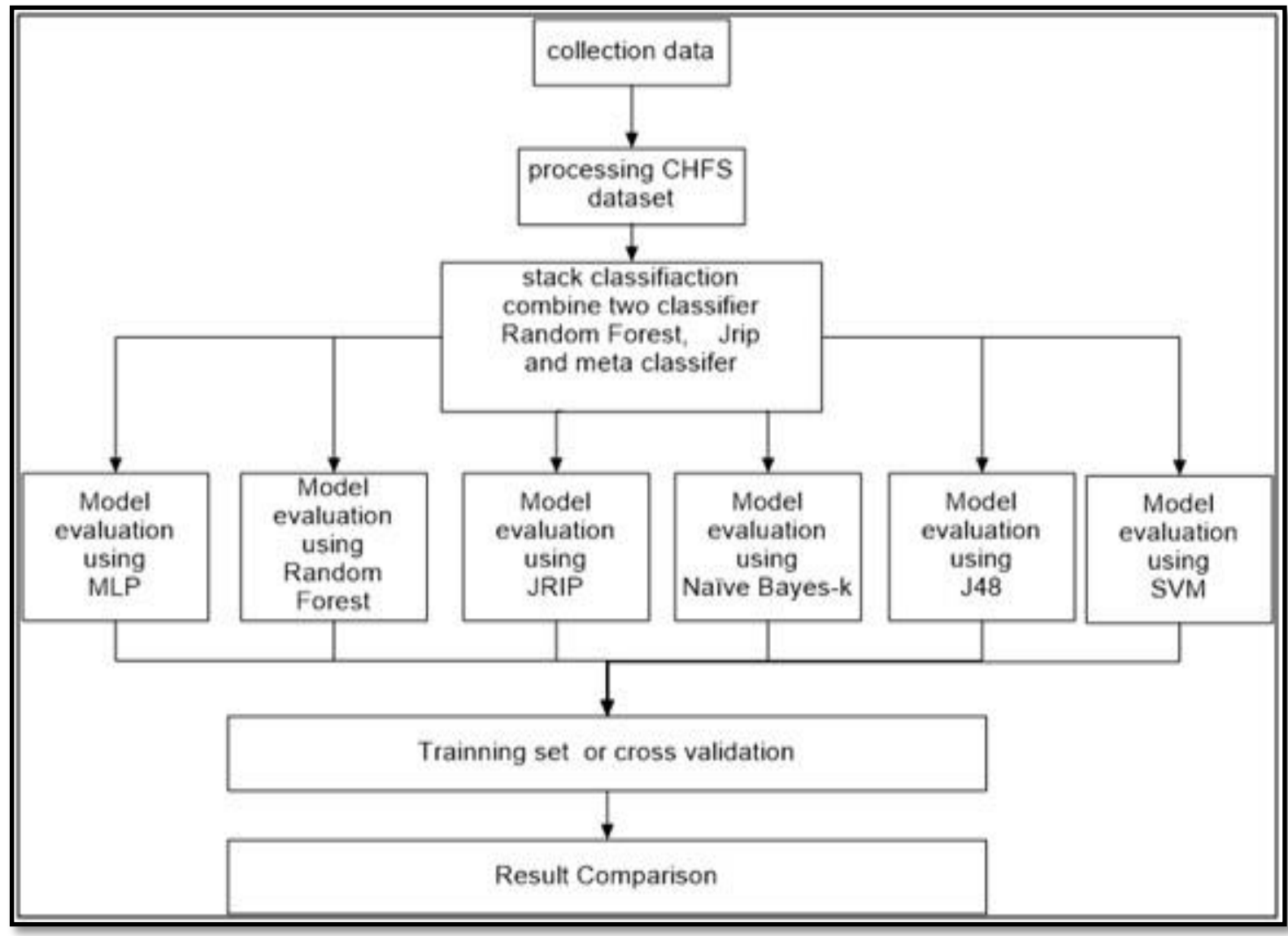

The author chooses permanent (Jrip, RF) based on a result from the table2, which achieves a good indicator of a fitness function problem.

Weka explorer is for exploring the data(weka,2019). Using explorer preprocessing can be performed for data. The result-oriented attribute can be selected, and the results can visualize. The experimenter used to understand the learning curve of stack hybrid classification based on (CHFS) and compare results with individual traditional classifiers without (CHFS). The knowledge flow interface lets the user represent learning algorithms and data sources into the required configuration. It enables the user to specify the data flow by connecting components representing data sources, pre-processing tools learning algorithms evaluation method, and visualization modules.

\subsection{Metrics used in health check systems for evaluation}

The different performance metrics generally used to explore the performance of the different models like sensitivity, accuracy, precision, and f-measure (Divya Jain \& Vijendra ,2017) . Accuracy: can be calculated by divide number of accurate predictions by the total number of all predications (Klöppel \& ...\&Frackowiak \& R. S,2008) 


$$
\text { Accuracy }=\frac{T N+T P}{f P+t P+f N+t N}
$$

Inside equation

$(\mathrm{FN}=$ False Negative, $\mathrm{FP}=$ False Positive, $\mathrm{TN}=$ True Negative, $\mathrm{TP}=$ True Positive $)$

\subsection{A Stack, Hybrid Classification Model, Based On Hybrid Feature Selection OF(MPEG7-Gabour filter-binary patterns pyramid - fuzzy 64 bin histogram) on MRI Dataset}

In below, figure 8 shows the architecture of all processes from feature extraction output will coming through the four image filter to extract the sensitive unseen data to prepare it into the classification process. Also, the classification process takes two ways of the test. The first way to consider the CNN approach in analyzing the MRI images and its major for extract and correctly classify the images with less error percentage depends on its algorithms. The other one we involved the data mining techniques and machine learning classifiers to in-depth into extracted data from four image filter in the feature extraction step. Besides, the comparison between Stack Hybrid classification with CNN classification.

Fig. 7 the proposed framework of stack hybrid classification on MRI images

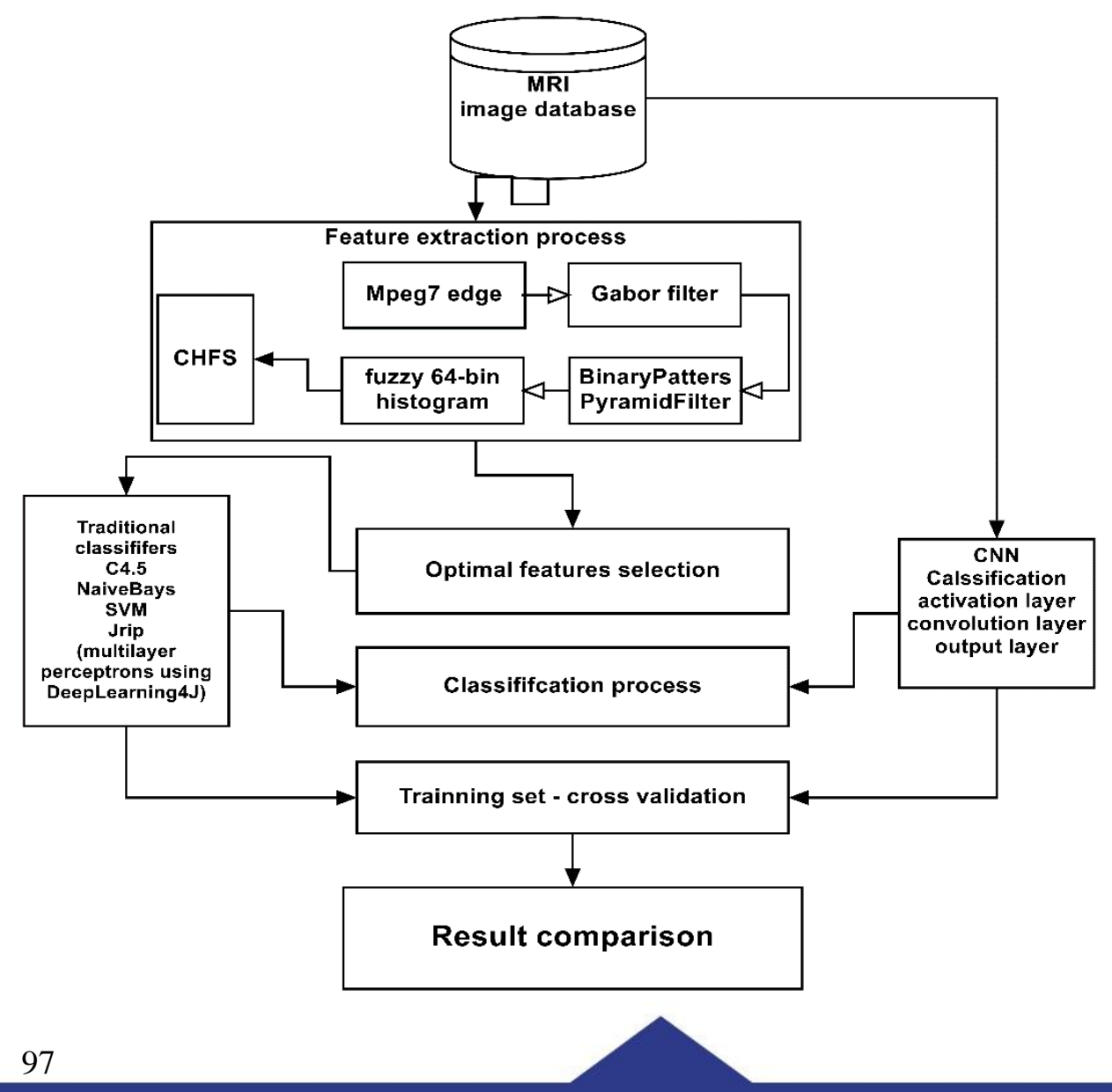




\section{Experimental Results and Evaluation}

The CSV file of the medical Dataset (clinical test) to patients of Alzheimer's dementia from oasis.org(online access Oasis Medical Dataset,2019) loaded to the weka tool. All experiments evaluated by the receiver operating characteristic (ROC) curve, accuracy, Fmeasure (Dina A. Ragab \& Omneya Attallah,2019).

\subsection{Result from proposed (CHFS) feature selection model on the clinical dataset}

Implement the dataset in the proposed model of CHFS, The input from the three feature selection methods,IG, GR, was applied for input to our optimization GA as initialization instead of randomly and set population size is 100 , number of generations is 20 , the crossover takes place at the middle position and mutation is prepared at one point randomly to reduce features of a dataset and extract the optimal feature subset and result was consisting of 4 features terms as shown in table 1.

Table 1:Number of feature extraction from three feature selection

\begin{tabular}{|c|c|c|}
\hline & Method & No.of Feature \\
\hline IG, GR & Filter & 6 \\
\hline GA & Wrapper & 6 \\
\hline $\begin{array}{c}\text { Optimized GA } \\
\text { Fitness Fun(C4.5) }\end{array}$ & Embedded & 4 \\
\hline
\end{tabular}

Source: (Weka open source software, 2018)

In table 2 below we calculate the fitness function of j48 and get accuracy with classical classifiers and repeat this procedure with other classifiers of calculation a fitness function too and obtain the accuracy for all, summerize result shown that the $\mathrm{j} 48$ is perform a good indicator as a fitness function with all classifiers. Furthermore, measure false-positive and false-negative for each chromosome. The chromosome, which has the lowest value, is considered an elite one(Divya Jain, Vijendra Singh,2018).

Table 2 Calculate the fitness function of optimizing Genetic Algorithm

\begin{tabular}{|c|c|c|c|c|c|}
\hline & FS(J48) & FS(NB) & FS(JR) & FS(RF) & FS(SVM) \\
\hline J48 & $73.06 \%$ & $69.41 \%$ & $72.50 \%$ & $69.81 \%$ & $72.57 \%$ \\
\hline NB & $75.91 \%$ & $80.79 \%$ & $72.41 \%$ & $72.89 \%$ & $70.87 \%$ \\
\hline MLP & $74.04 \%$ & $68.84 \%$ & $72.58 \%$ & $68.83 \%$ & $72.57 \%$ \\
\hline RF & $74.04 \%$ & $77.71 \%$ & $72.66 \%$ & $77.54 \%$ & $72.66 \%$ \\
\hline SVM & $72.57 \%$ & $68.83 \%$ & $72.23 \%$ & $68.82 \%$ & $72.82 \%$ \\
\hline JRIP & $72.90 \%$ & $70.13 \%$ & $74.85 \%$ & $75.34 \%$ & $74.85 \%$ \\
\hline
\end{tabular}

The threshold value equal to 0.02 chosen for information gain, as shown in Fig 10, lead to reduce feature term to 5 attributes, and a value greater than or equal to 0.04 selected for gain ratio method to rearrange the feature term as priority and preparation for classification method to obtain the maximum efficiency. Table 1 discusses the results of all the techniques. If 10fold cross-validation applied. For summarized results in the table (3) from the weka software 
of the analytics dataset, we used the (ROC) curve to evaluate the output result from our proposed architecture model, as shown in fig 8,9.

Table 3: Experimental result of Accuracy pre-post CHFS model with various classifiers

\begin{tabular}{|c|c|c|}
\hline Classifier & $\begin{array}{c}\text { Pre- (CHFS) Feature } \\
\text { Selection Accuracy }\end{array}$ & $\begin{array}{c}\text { Post }- \text { (CHFS) Feature } \\
\text { Selection Accuracy }\end{array}$ \\
\hline J48 & $\mathbf{7 1 . 9 2 \%}$ & $\mathbf{7 3 . 0 6 \%}$ \\
\hline SVM & $\mathbf{6 8 . 8 3 \%}$ & $\mathbf{7 2 . 5 7 \%}$ \\
\hline Nä̈ve Bayes-k & $\mathbf{6 5 . 1 7 \%}$ & $\mathbf{7 5 . 9 1 \%}$ \\
\hline JRIP & $\mathbf{7 2 . 5 7 \%}$ & $\mathbf{7 2 . 9 0 \%}$ \\
\hline Random Forest & $\mathbf{7 8 . 2 7 \%}$ & $\mathbf{7 4 . 0 4 \%}$ \\
\hline $\begin{array}{c}\text { Multiyear } \\
\text { perceptron }\end{array}$ & $\mathbf{6 8 . 8 3 \%}$ & $\mathbf{7 4 . 3 6 \%}$ \\
\hline
\end{tabular}

Fig.8 Summarized accuracy results of pre-post (CHFS) feature selection

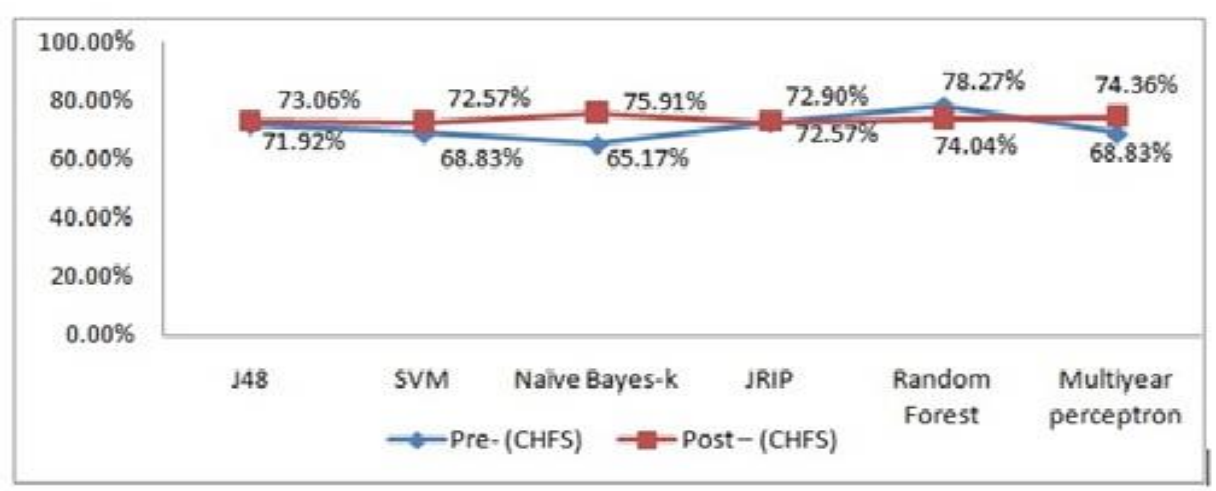

Source: (Weka open source software, 2018)

Fig.9ROC curve and precision-recall curve for all six classifiers on raw features (a) Pre-CHFS model

(b) Post-CHFS model (c) Precision and recall curve pre-CHFS model(d) Precision and recall curve postCHFS model

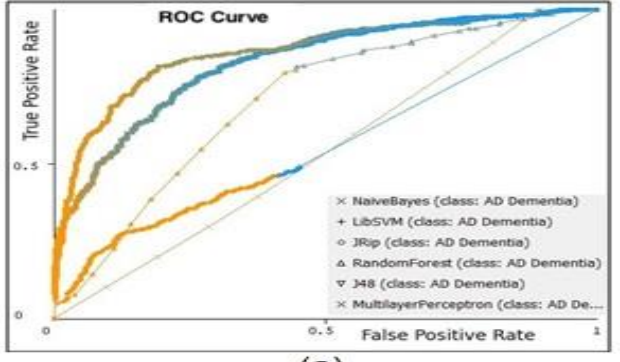

(a)

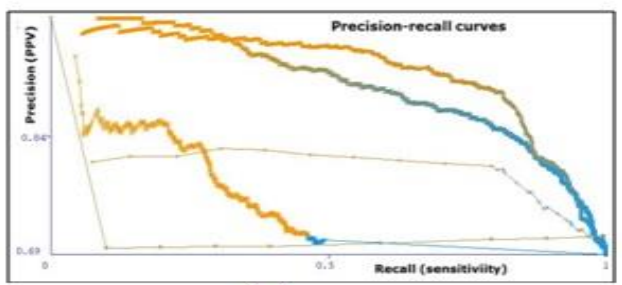

(c)

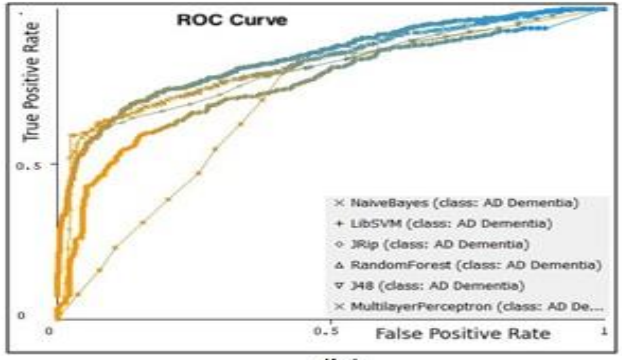

(b)

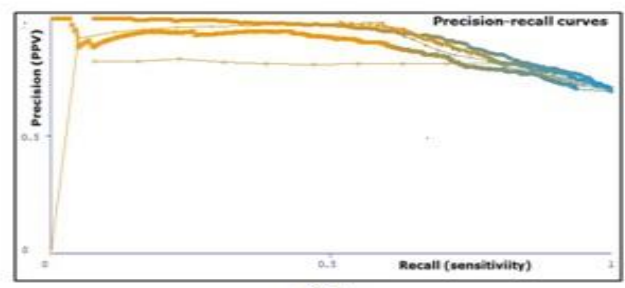

(d)

Source: (Weka open source software, 2018) 
As an essential point, the proposed (CHFS) model compared against a popular dimensionality reduction technique, the Principal Component Analysis (PCA) (Joliffe IT,2002) . We have used PCA to reduce the number of variables of our oasis medical dataset(online access Oasis Medical Dataset,2019), and the result was three features PCA selected at $91.1 \%$ accuracy of variance and five features of PCA selected at $94.3 \%$ accuracy. The result of this comparison between PCA and our proposed CHFS feature selection model with six classifiers, as shown in Table 4.Furthermore, summarized in fig. 10, Fig 11, explained the ROC curve to evaluate the result of this comparison of our feature selection technique evaluation.

Table 4: Summary of 10-fold Cross-Validation (CHFS)Model Accuracy Compared Against PCA

\begin{tabular}{|c|c|c|c|}
\hline \multirow{2}{*}{ Classifier } & \multicolumn{2}{|c|}{ PCA Accuracy } & (CHFS) Model \\
\cline { 2 - 3 } & $\begin{array}{c}\text { Components } \\
(91.1 \%)\end{array}$ & $\begin{array}{c}\text { (5) components } \\
(94.3 \%)\end{array}$ & $\begin{array}{c}\text { Feature Selection } \\
\text { Accuracy }\end{array}$ \\
\hline J48 & $68.72 \%$ & $68.83 \%$ & $73.06 \%$ \\
\hline SVM & $68.72 \%$ & $68.80 \%$ & $72.57 \%$ \\
\hline Naïve Bayes & $72.78 \%$ & $72.74 \%$ & $75.91 \%$ \\
\hline JRIP & $69.73 \%$ & $69.81 \%$ & $72.90 \%$ \\
\hline Random Forest & $72.74 \%$ & $72.8 \%$ & $74.04 \%$ \\
\hline Multiyear.perceptron & $69.43 \%$ & $69.83 \%$ & $74.36 \%$ \\
\hline
\end{tabular}

Fig.10 Summarized accuracy results of PCA versus (CHFS) feature selection

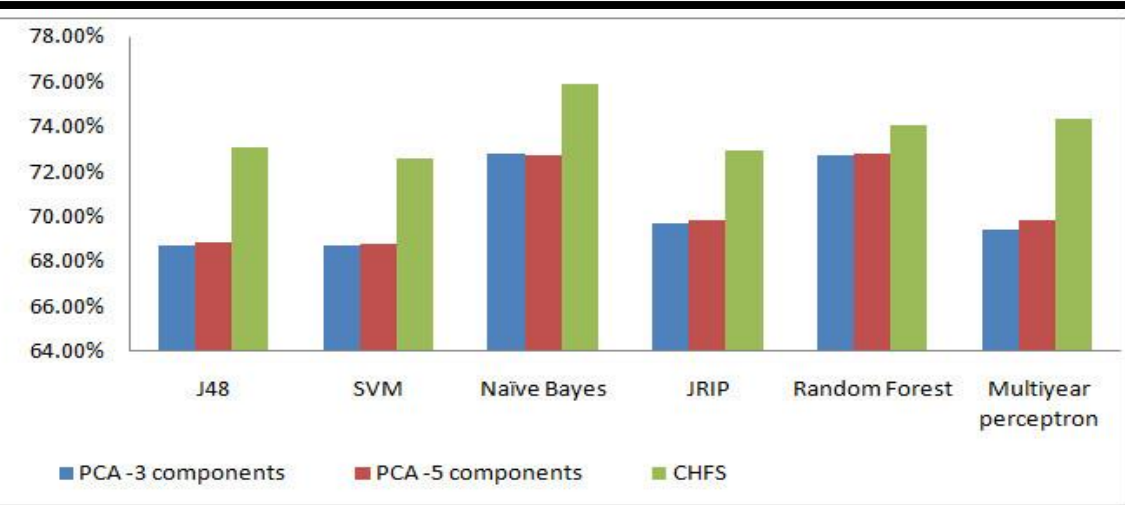

Fig.11 ROC curve for all six classifiers on raw features (a) Roc of a medical dataset on traditional classifiers (b) Of 3-component-PCA(c) Of 5- component-PCA (d) Post-CHFS model(d) Post-CHFS model 


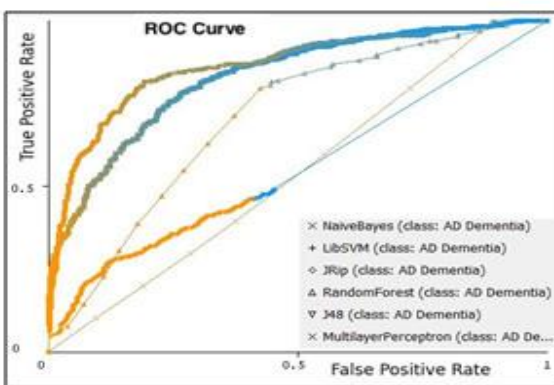

(a)

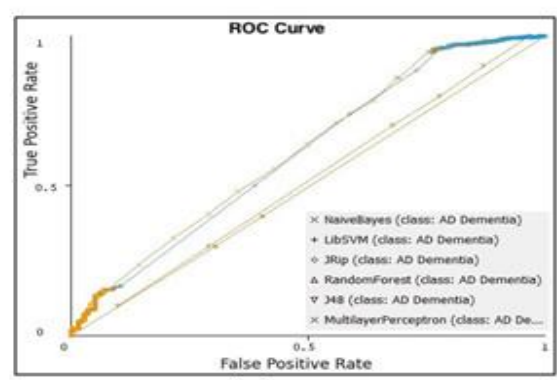

(c)

Source: (Weka open source software, 2018)

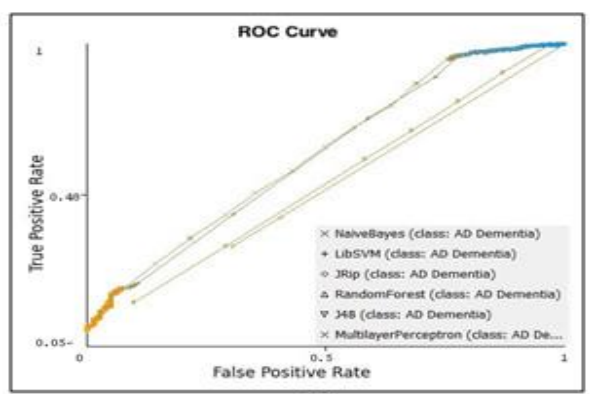

(b)

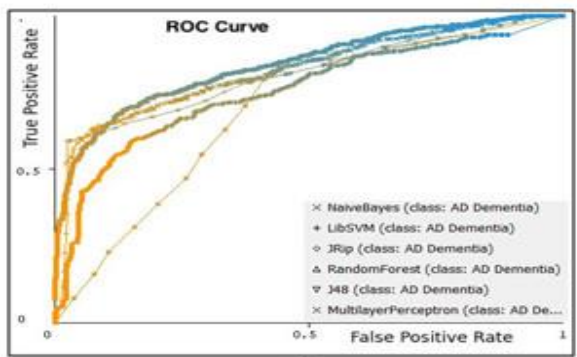

(d)

\subsection{Result of proposed Stack Hybrid Classification Model Based on (CHFS) Feature Selection on a clinical dataset}

The classification techniques applied to medical dataset. (Yasser Fouad \&...\& Hatem Khater,2015) . Can be achieved by

$$
\text { ClassificationAccuracy }=\frac{\text { CorrectlyClassifiedSamples }}{\text { TotalNumberofSamples }} * 100
$$

The authors use the output dataset from CHFS feature selection to run it inside the proposed classification model to flow knowledge in the weka area tool and begin the combination process of random forest and Jrip classifier with six classifiers as a metaclassifier individually.

\subsubsection{Hybrid classification combination process of (random forest, Jrip) with j48 stack Meta classifier}

The result of non-Alzheimer's samples showed that predicted to be infected with Alzheimer's disease and high overall accuracy $(89.34 \%)$ compared with the j48 classifier individually (71.92\%).

Fig. 12 Confusion matrix and ROC curve for J48 classifier on raw features (a) Confusion matrix Pre-Hybrid classification model Fig 18. (b) Confusion matrix Post- Hybrid classification modeling 18. (c) ROC curve pre-

Hybrid classification modeling 18. (d) ROC curve post- Hybrid classification model 


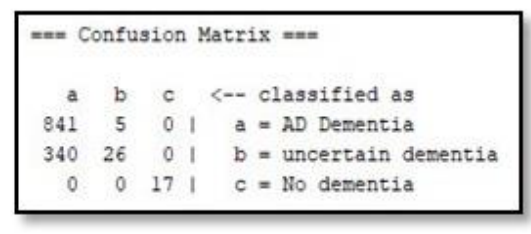

(a)

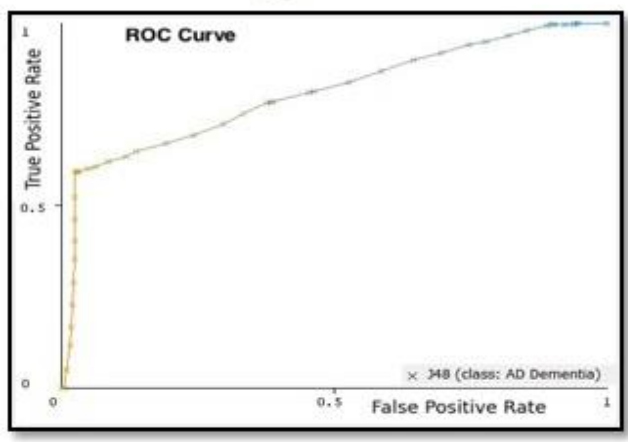

(c)

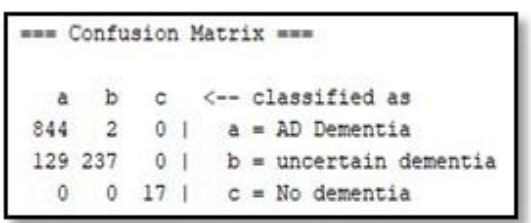

(b)

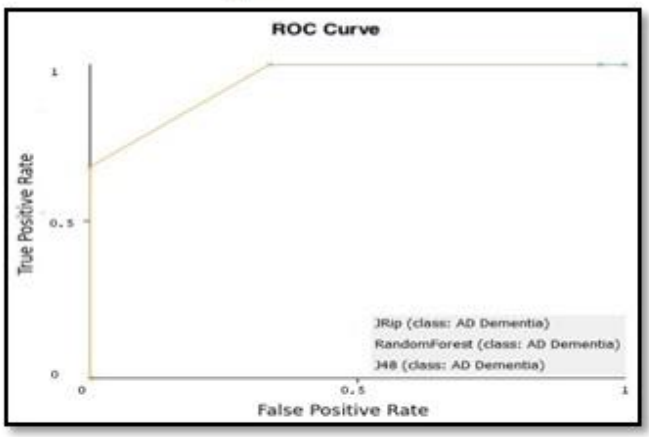

(d)

Source: (Weka open source software, 2018)

\subsubsection{Hybrid classification of (random forest, jrip) with SVMmeta-classifier}

The result was a high overall accuracy (96.50\%) compared with the SVM classifier individually $(68.83 \%)$, as shown in fig 13.

Fig 13. Confusion matrix and ROC curve for SVM classifier on raw features (a) Confusion matrix Pre-Hybrid classification model (b) Confusion matrix Post-Hybrid classification model(c) ROC curve pre-Hybrid classification model (d) ROC curve post- Hybrid classification model

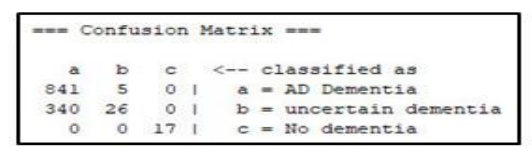

(a)

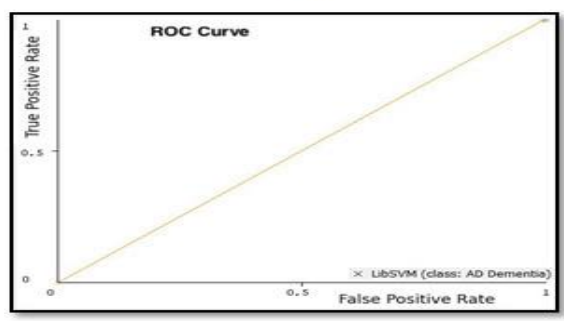

(c)

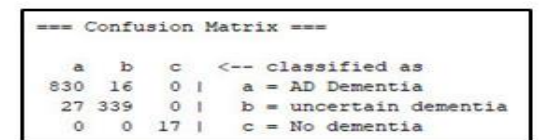

(b)

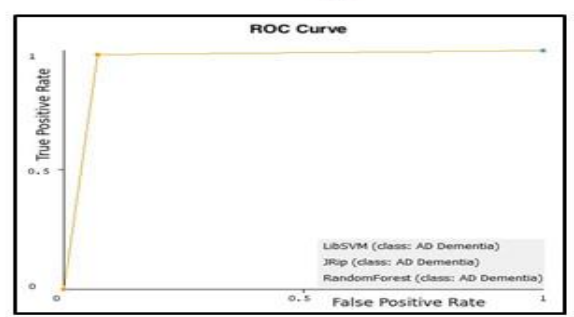

(d)

Source: (Weka open source software, 2018)

\subsubsection{Hybrid classification of (random forest, jrip) with Naive Bayesas meta-classifier}

The result is a high overall accuracy (89.09\%) compared with Naive Bayes classifier individually $(65.17 \%)$, as shown in fig 14 . 
Fig.14 Confusion matrix and ROC curve for Naive Bayes classifier on raw features (a) Confusion matrix Pre-Hybrid classification model (b) Confusion matrix Post-Hybrid classification model (c)curve pre-Hybrid classification model(d) ROC curve post-Hybrid classification model

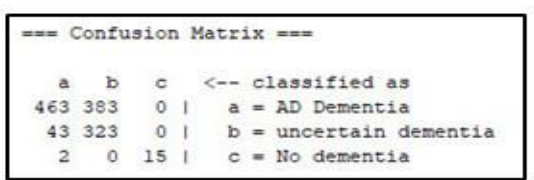

(a)

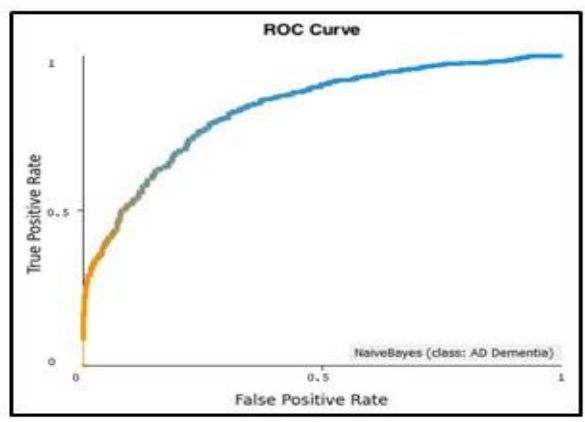

(c)

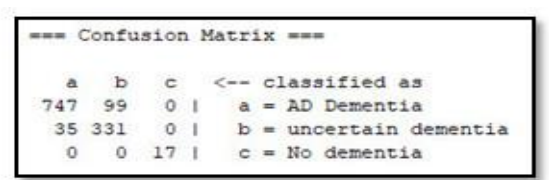

(b)

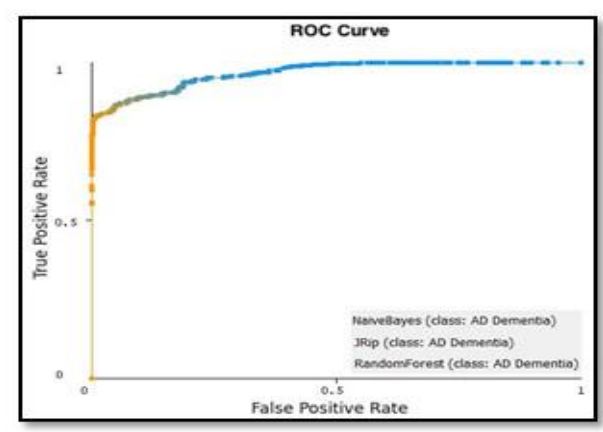

(d)

Source: (Weka open source software, 2018)

\subsubsection{Hybrid classification of (random forest, Jrip) with Jripas meta-classifier}

the result is a high overall accuracy (85.59\%) compared with Naive Bayes classifier individually $(72.57 \%)$, as shown in fig 15

Fig.15Confusion matrix and ROC curve for Jrip classifier on raw features (a) Confusion matrix Pre-Hybrid classification model (b) Confusion matrix Post-Hybrid classification model (c) ROC curve pre- Hybrid classification model(d) ROC curve post- Hybrid classification mode

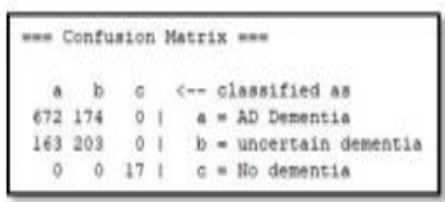

(a)

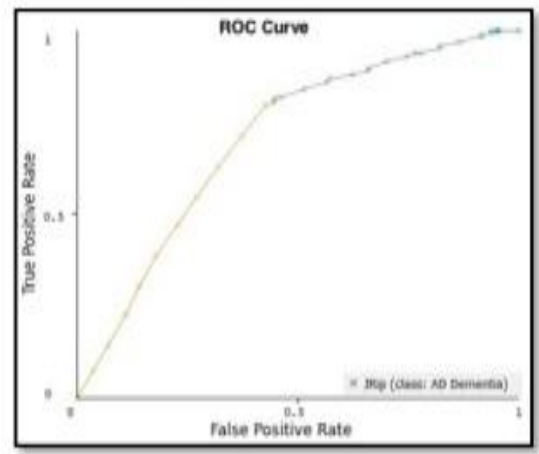

(c)

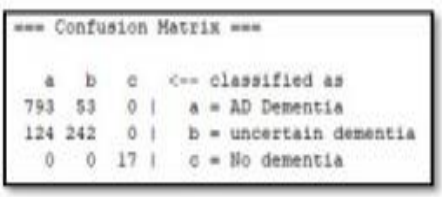

(b)

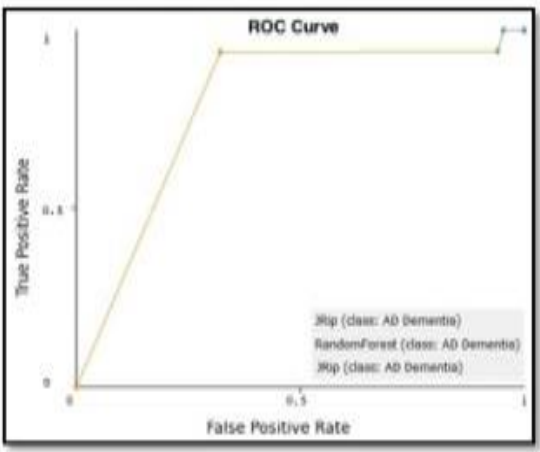

(d)

Source: (Weka open source software, 2018) 


\subsubsection{Hybrid classification of (random forest, jrip) with a random forest as meta- classifier}

The result is a high overall accuracy $(80.71 \%)$ compared with random forest classifiers individually (78.27\%), as shown in fig 16.

Fig.16 Confusion matrix and ROC curve for random forest classifier on raw features (a) Confusion matrix Pre-Hybrid classification model(b) Confusion matrix Post-Hybrid classification model (c) ROC curve preHybrid classification model(d) ROC curve post- Hybrid classification model

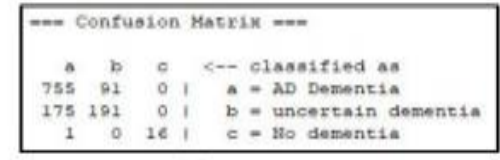

(a)

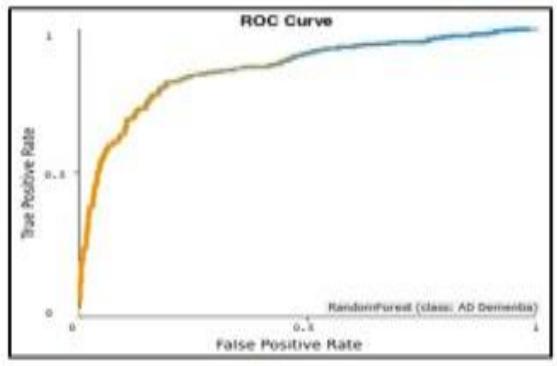

(c)

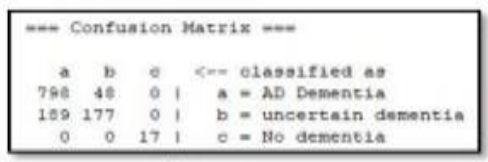

(b)

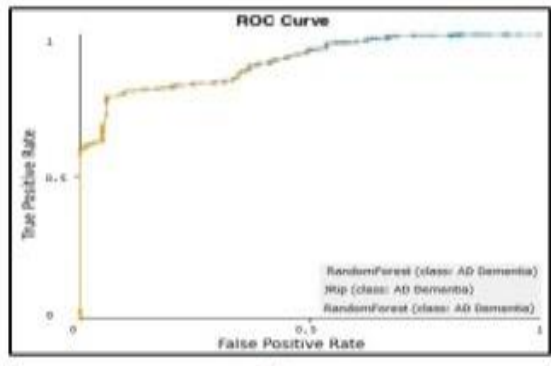

(d)

Source: (Weka open source software, 2018)

\subsubsection{Hybrid classification of (random forest, jrip) with Multilayer Perceptron as meta- classifier}

The result is a high overall accuracy of (83\%) compared with the Multilayer Perceptron classifier individually of $(68.83 \%)$ in training set mode, as shown in fig 17.

Fig.17 Confusion matrix and ROC curve for Multilayer Perceptron classifier on raw features (a) Confusion matrix Pre-Hybrid classification model (b) Confusion matrix Post-Hybrid classification model(c) ROC curve pre-Hybrid classification model(d) ROC curve post-Hybrid classification model

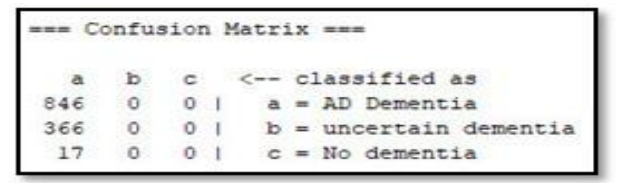

(a)

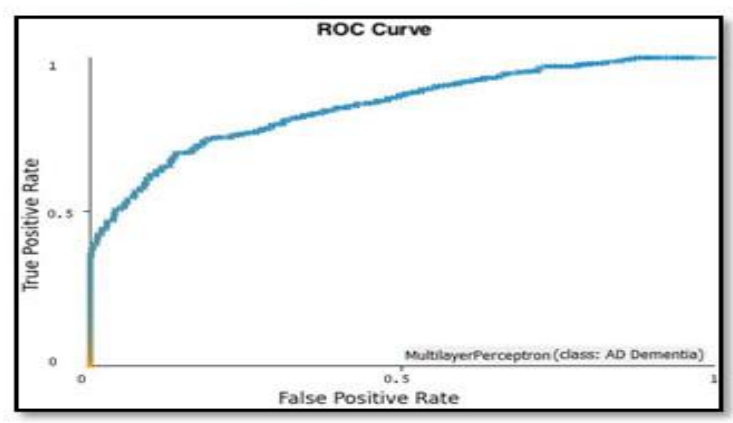

(c)

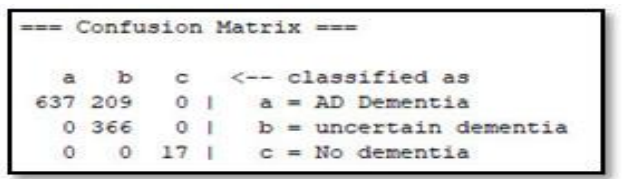

(b)

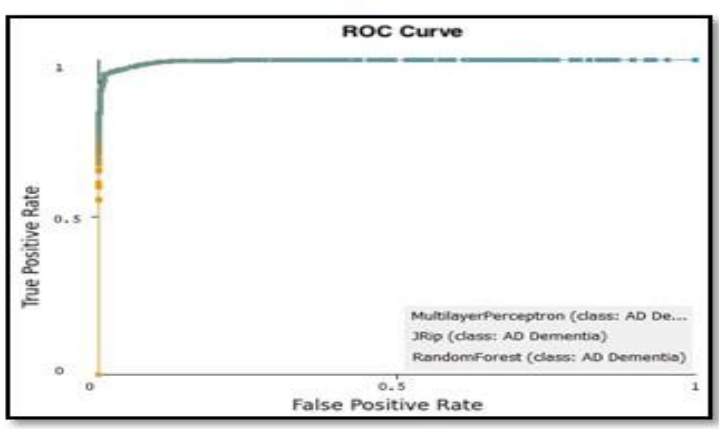

(d) 
Source: (Weka open source software, 2018)

\subsection{Applying Convolution neural network (CNN) on MRI images}

The author's aims to improve clinical data for early diagnose of $\mathrm{AD}$ and to prove that we make a comparison between clinical data diagnose and MRI diagnoses in Early-stage, We tested a set of Alzheimer's MRI images from the kaggle.com benchmark web of dataset science, and the dataset contains 5121 MRI image divided to 4 classes(mild, very mild,non, moderate) as shown in below figure 16 to compare with clinical data to perform the accuracy of early-disease diagnosis by the convolution neural network as shown in the below layout model in figure 18

Figure 18 the types of MRI classes for AD disease on the brain

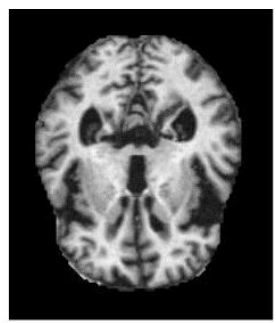

Mild

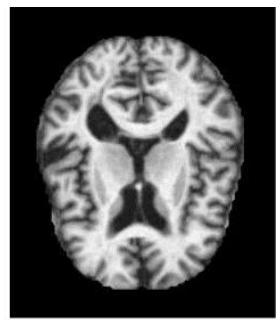

Moderate

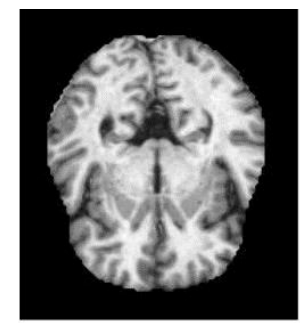

Non-dementia

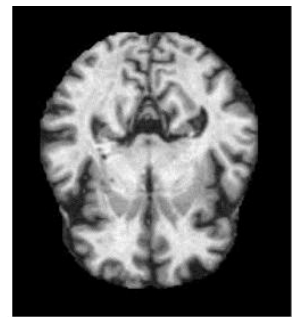

Very Mild

Figure 19 shows the prediction model for MRI images
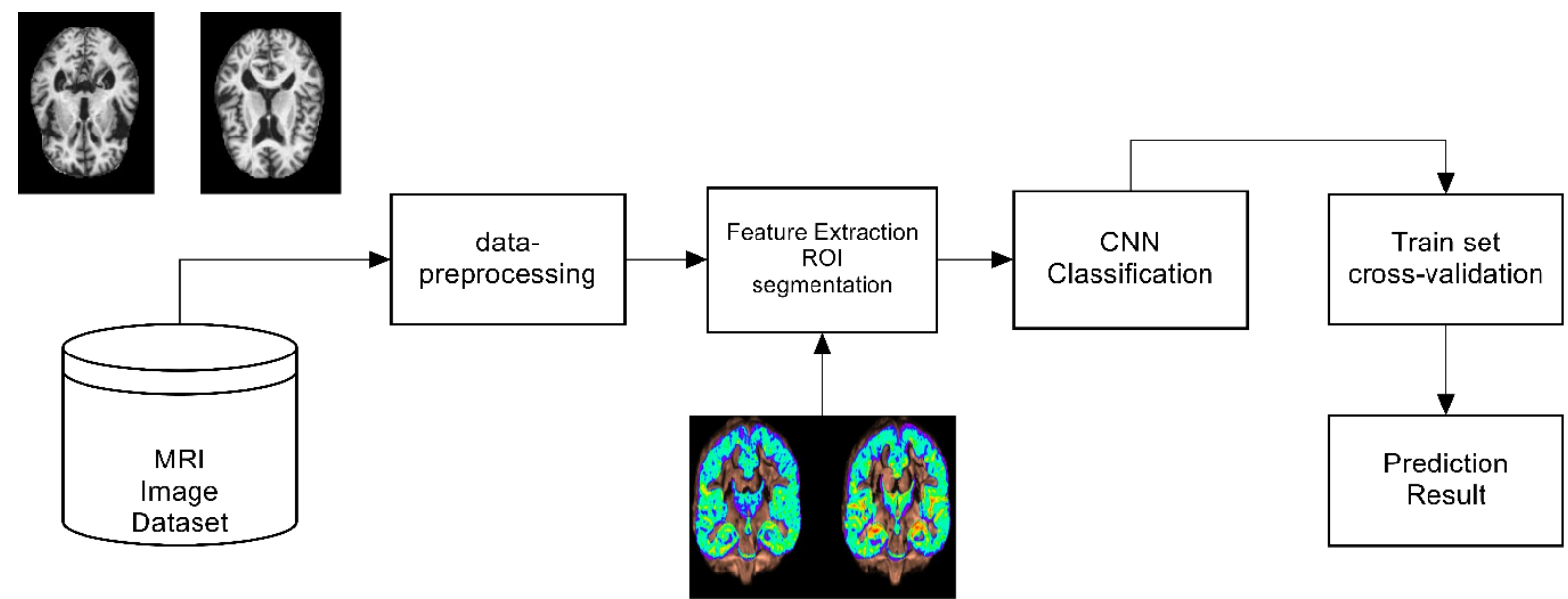

In the below figure 20, we propose the result of MRI classification on Kaggle 5121 images dataset by using the convolution neural network and the result evaluated by ROC curve $-\mathrm{f}$ measure 
Fig.20 ROC curve of MRI category and confusion matrix of CNN classification
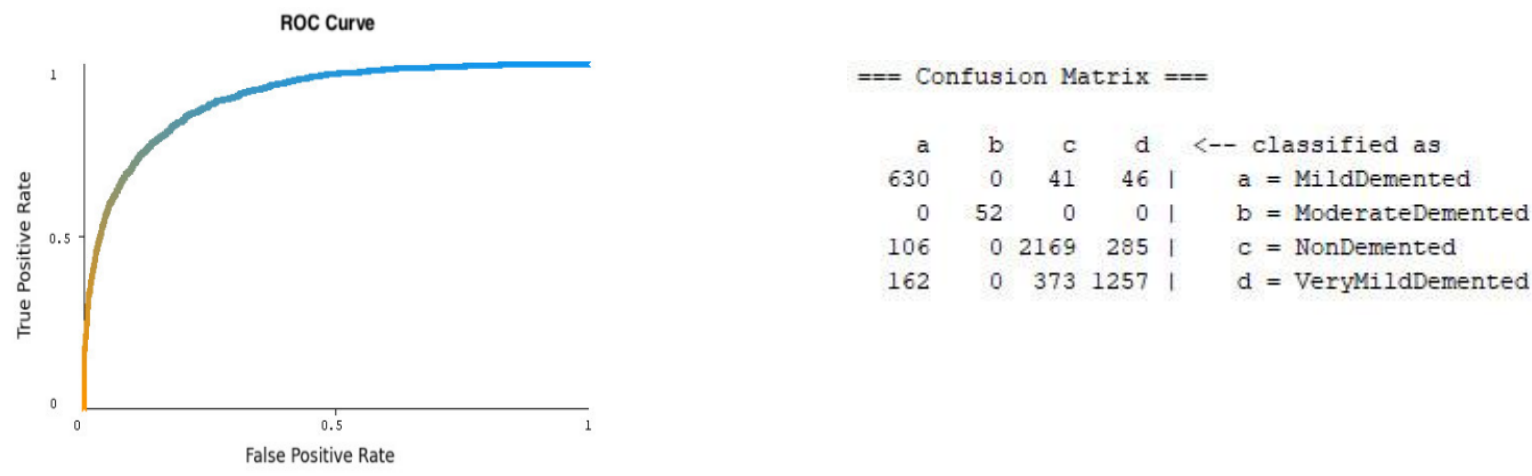

And in the below figure 21 shown the error curve of CNN classification.

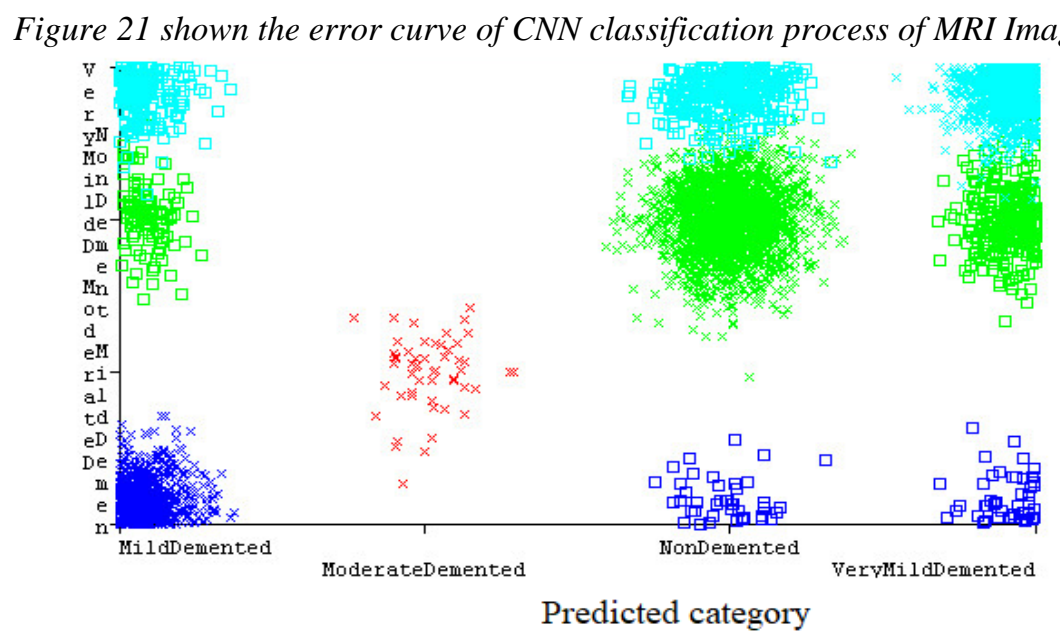

Table 5 The details of result from CNN-MRI Model

\begin{tabular}{|c|c|c|c|}
\hline F-measure & TPR & ROC & PRC \\
\hline $80.1 \%$ & $80.2 \%$ & $92.6 \%$ & $89.2 \%$ \\
\hline
\end{tabular}

The classification accuracy of the convolution neural network (CNN) was $80.21 \%$ and $\mathrm{f}$ measure $80.1 \%$, which considered least than the classification accuracy of our clinical data, which confirms the importance of our proposed feature extraction and hybrid classification of producing CHFS-SVM model of $96.5 \%$ in early diagnoses. 


\subsection{Applying a Novel Hybrid Feature selection model and Stack Hybrid classification on MRI images}

The MRI images dataset collected from Kaggle database website with a total of 5121 images each segregated into the severity of Alzheimer's, first we apply four image filter mention in the proposed architecture above in fig 6 on MRI images to extract the mathematical data analysis relation to applying the CHFS model and the result of feature extracted shown below in table6.

Table 6:Number of feature extraction from CHFS feature selection model
\begin{tabular}{|c|c|c|}
\hline & Method & No.of Feature \\
\hline MPEG-7 & Image filter & 82 \\
\hline Gabor & Image filter & 142 \\
\hline $\begin{array}{c}\text { Binary patterns } \\
\text { pyramid }\end{array}$ & Image filter & 898 \\
\hline $\begin{array}{c}\text { fuzzy 64 bin } \\
\text { histogram }\end{array}$ & Image filter & 1473 \\
\hline IG, GR & Filter & 182 \\
\hline GA & Wrapper & 134 \\
\hline $\begin{array}{c}\text { Optimized GA } \\
\text { Fitness Fun(C4.5) }\end{array}$ & Embedded & 44 \\
\hline
\end{tabular}

Besides using four image filters to extract the features from MRI images, which extract 82 features from MPEG-7 filter and 142 features after applying Gabor filter with 898 features from binary patterns pyramid with final applying of fuzzy 64-bin histogram to produce total 1473 features from MRI dataset. Besides, applying our proposed CHFS with optimization of genetic algorithm to get the final 44 sensitive features.

Table 6 discusses the results of all the techniques. If 10- fold cross-validation applied. For summarized results in the table (65) from the weka software of the analytics dataset, we used the (ROC) curve to evaluate the output result from our proposed architecture model, as shown in fig 22 , table 7 .

Table 7: Experimental result of Accuracy pre-post CHFS model with various classifiers on MRI images

\begin{tabular}{|c|c|c|}
\hline Classifier & $\begin{array}{c}\text { Pre- (CHFS) Feature } \\
\text { Selection Accuracy } \\
\text { By using four image } \\
\text { filter with 1473 feature }\end{array}$ & $\begin{array}{c}\text { Post - (CHFS) Feature } \\
\text { Selection Accuracy }\end{array}$ \\
\hline SVM & $\mathbf{5 4 . 9 3 \%}$ & $\mathbf{6 1 . 2 7 \%}$ \\
\hline Naïve Bayes & $\mathbf{4 8 . 6 6 \%}$ & $\mathbf{4 2 . 7 0 \%}$ \\
\hline JRIP & $\mathbf{5 2 . 7 8 \%}$ & $\mathbf{5 6 . 7 8 \%}$ \\
\hline Random Forest & $\mathbf{6 1 . 9 7 \%}$ & $\mathbf{8 6 . 1 5 \%}$ \\
\hline
\end{tabular}


The overall improvement in our experiment on traditional classifiers produce $22.10 \%$ after using our proposed feature extraction from MRI dataset images. The figure below shows it summarize for all 3 for all results in table 6 .

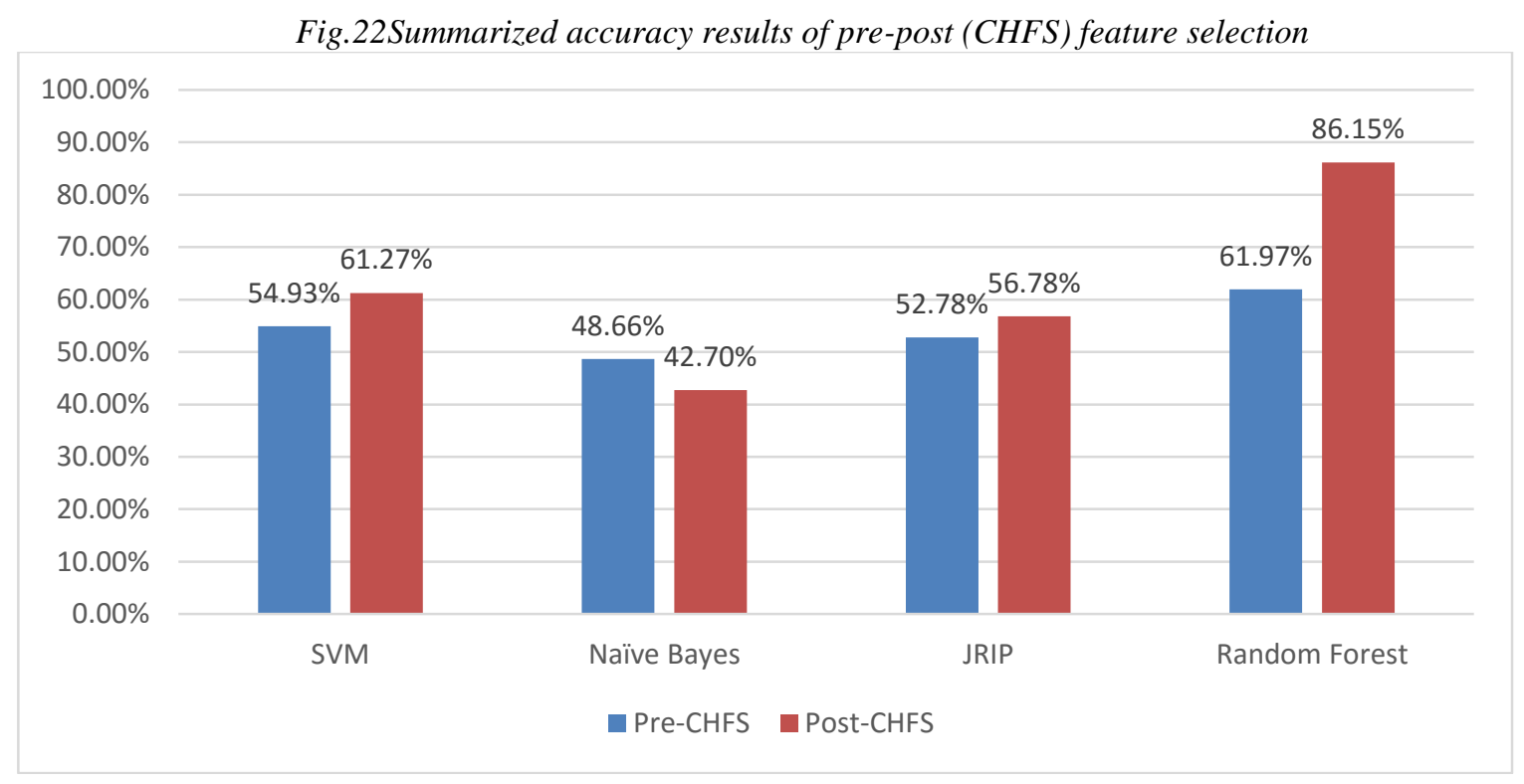

\subsection{Improved MRI Classification by Applying a proposed Stack Hybrid Classification Model Based on Proposed CHFS Feature Extraction Method}

The author applying a novel stack hybrid classification as described above in section 4.3 on the MRI dataset to improve the classification accuracy of images by using a traditional algorithms of classifiers but differently as a combination process of(Jrip, Random Forest)classifiers with stacked meta classifiers of (Naive Bayes-SVM-Jrip-Random Forest). In table 8 , the result of the comparison of accuracy between pre-post applying our proposed classification model based on the CHFS feature extraction model and in figure 23,24, the ROC curve of all classifiers process with the result of accuracy and f-measure precisionrecall-true positive rate-false positive rate for our proposed model as shown in table 8 .

Table 8: Experimental result of Accuracy pre-post Stack Hybrid model with various classifiers on MRI

\begin{tabular}{|c|c|c|}
\hline \multirow{2}{*}{ Classifier } & $\begin{array}{c}\text { images } \\
\text { Accuracy } \\
\text { Pre- Stack Hybrid } \\
\text { (POST-CHFS) }\end{array}$ & $\begin{array}{c}\text { Accuracy } \\
\text { Post - Stack Hybrid- } \\
\text { (POST-CHFS) }\end{array}$ \\
\hline SVM & $\mathbf{6 1 . 2 7 \%}$ & $\mathbf{9 8 . 6 1 \%}$ \\
\hline Naïve Bayes & $\mathbf{4 2 . 7 0 \%}$ & $\mathbf{9 7 . 6 3 \%}$ \\
\hline JRIP & $\mathbf{5 6 . 7 8 \%}$ & $\mathbf{9 4 . 4 9 \%}$ \\
\hline Random Forest & $\mathbf{8 6 . 1 5 \%}$ & $\mathbf{9 8 . 9 9 \%}$ \\
\hline
\end{tabular}


Figure 23 The summerize result of accuracy pre-post proposed Stack Hybrid Classification

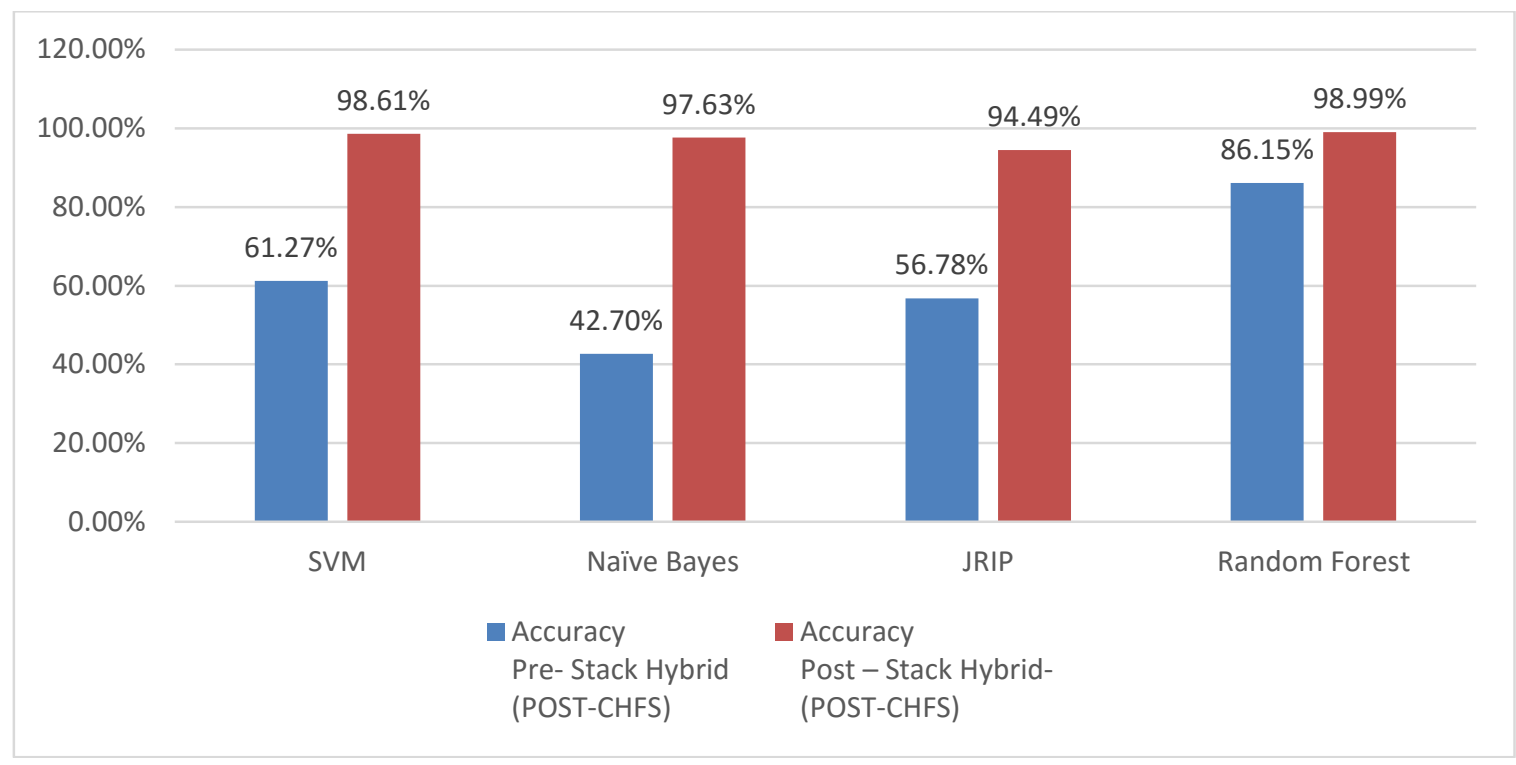

Fig.24 ROC curve for pre-post stack hybrid classifications based on CHFS

(a) ROC curve pre-Hybrid classification model

(b) ROC curve post- Hybrid classification model

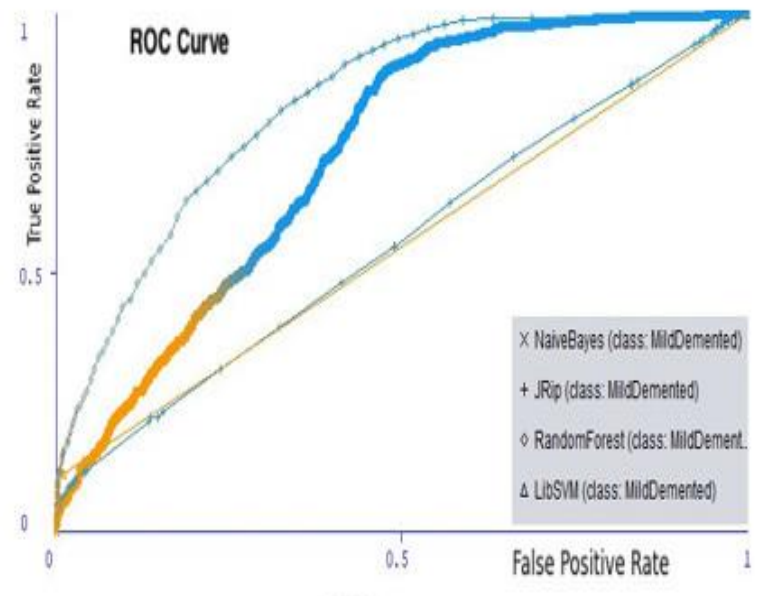

(a)

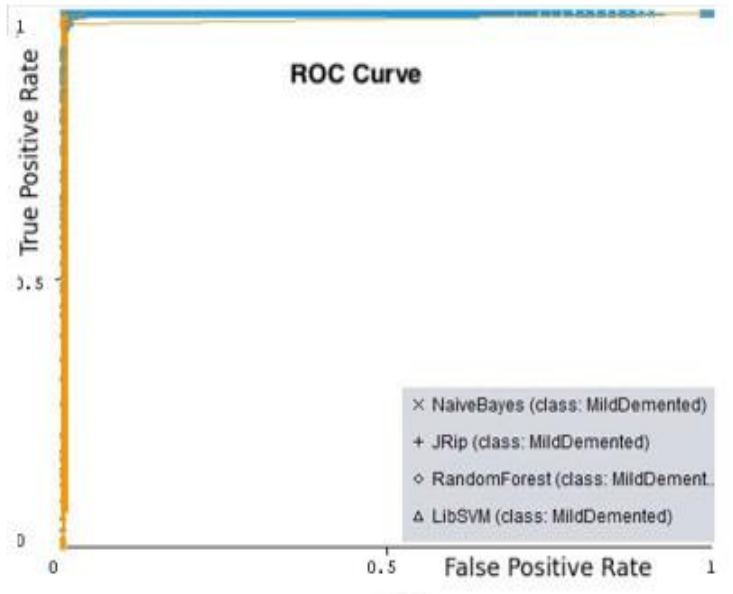

(b)

Table 9. The avg. detailed accuracy for MRI-dataset class of Stacked hybrid classification

\begin{tabular}{|c|c|c|c|c|c|c|c|}
\hline class & TP Rate & FP Rate & Precision & Recall & F-Measure & ROC Area & PRC Area \\
\hline MildDemented & 0.999 & 0.000 & 1.000 & 0.999 & 0.999 & 1.000 & 1.000 \\
\hline ModerateDemented & 0.135 & 0.000 & 1.000 & 0.135 & 0.237 & 1.000 & 0.975 \\
\hline NonDemented & 0.999 & 0.006 & 0.994 & 0.999 & 0.996 & 1.000 & 1.000 \\
\hline VeryMildDemented & 1.000 & 0.010 & 0.981 & 1.000 & 0.991 & 1.000 & 1.000 \\
\hline Avg. & 0.990 & 0.007 & 0.991 & 0.990 & 0.987 & 1.000 & 1.000 \\
\hline
\end{tabular}




\section{Discussion}

The authors compare the results of the proposed feature extraction model and stack hybrid classification on clinical data and neuroimaging dataset in different cases pre-post feature extraction and pre-post proposed hybrid classification and the result compared with $\mathrm{CNN}$ MRI model on images dataset. These comparisons, according to our proposed model presented in this study, were reduced a false negative rate and showed a relatively high overall accuracy with more accurate results. The best result extracted from our proposed model was CHFS-Stacked (jrip, RF) with SVM classifier accuracy of 96.5\% compared to the output result of CNN-MRI accuracy of $80.21 \%$. After that, the author applying a novel feature extraction on MRI images with four image filters later with the CHFS model of optimizing the GA algorithm to best select sensitive features from MRI images. The reduction of selected features from 1473 to 44 features. In the next stage, we applying our proposed stack hybrid classification on 44 features among four different classifiers to obtain the improves in the classification process with reaching to $98.99 \%$ improving better than CNN-MRI model, as shown in fig 19, and table 10.

Table 10: Summary of 10-fold Cross-validation of Stack Hybrid Classification Based on (CHFS) In Weka3.9

\begin{tabular}{|c|c|c|c|c|c|c|}
\hline \multirow[b]{2}{*}{$\begin{array}{l}\text { Traditional } \\
\text { Classifier }\end{array}$} & \multicolumn{3}{|c|}{ Clinical Dataset } & \multicolumn{3}{|c|}{ MRI Dataset } \\
\hline & $\begin{array}{c}\text { Accuracy } \\
\text { Pre } \\
(\mathrm{CHFS})\end{array}$ & $\begin{array}{c}\text { Accuracy } \\
\text { post } \\
(\mathrm{CHFS})\end{array}$ & $\begin{array}{c}\text { Accuracy } \\
\text { Post- CHFS- } \\
\text { stack Hybrid } \\
\text { classification }\end{array}$ & $\begin{array}{c}\text { Accurac } \\
y \\
\text { Pre }(\mathrm{CHFS})\end{array}$ & $\begin{array}{c}\text { Accuracy } \\
\text { post (CHFS) }\end{array}$ & $\begin{array}{c}\text { Accuracy } \\
\text { Post-CHFS- } \\
\text { stackHybrid } \\
\text { classification }\end{array}$ \\
\hline SVM & $68.83 \%$ & $72.57 \%$ & $96.50 \%$ & $54.93 \%$ & $61.27 \%$ & $98.61 \%$ \\
\hline $\begin{array}{l}\text { Naive } \\
\text { Bayes }\end{array}$ & $65.17 \%$ & $75.91 \%$ & $89.09 \%$ & $48.66 \%$ & $42.70 \%$ & $97.63 \%$ \\
\hline JRIP & $72.57 \%$ & $72.90 \%$ & $85.59 \%$ & $52.78 \%$ & $56.78 \%$ & $94.49 \%$ \\
\hline $\begin{array}{c}\text { Random } \\
\text { Forest }\end{array}$ & $78.27 \%$ & $74.04 \%$ & $80.71 \%$ & $61.97 \%$ & $86.15 \%$ & $98.99 \%$ \\
\hline
\end{tabular}

Fig.19 Summarized results of pre-post stack classification based on (CHFS) 


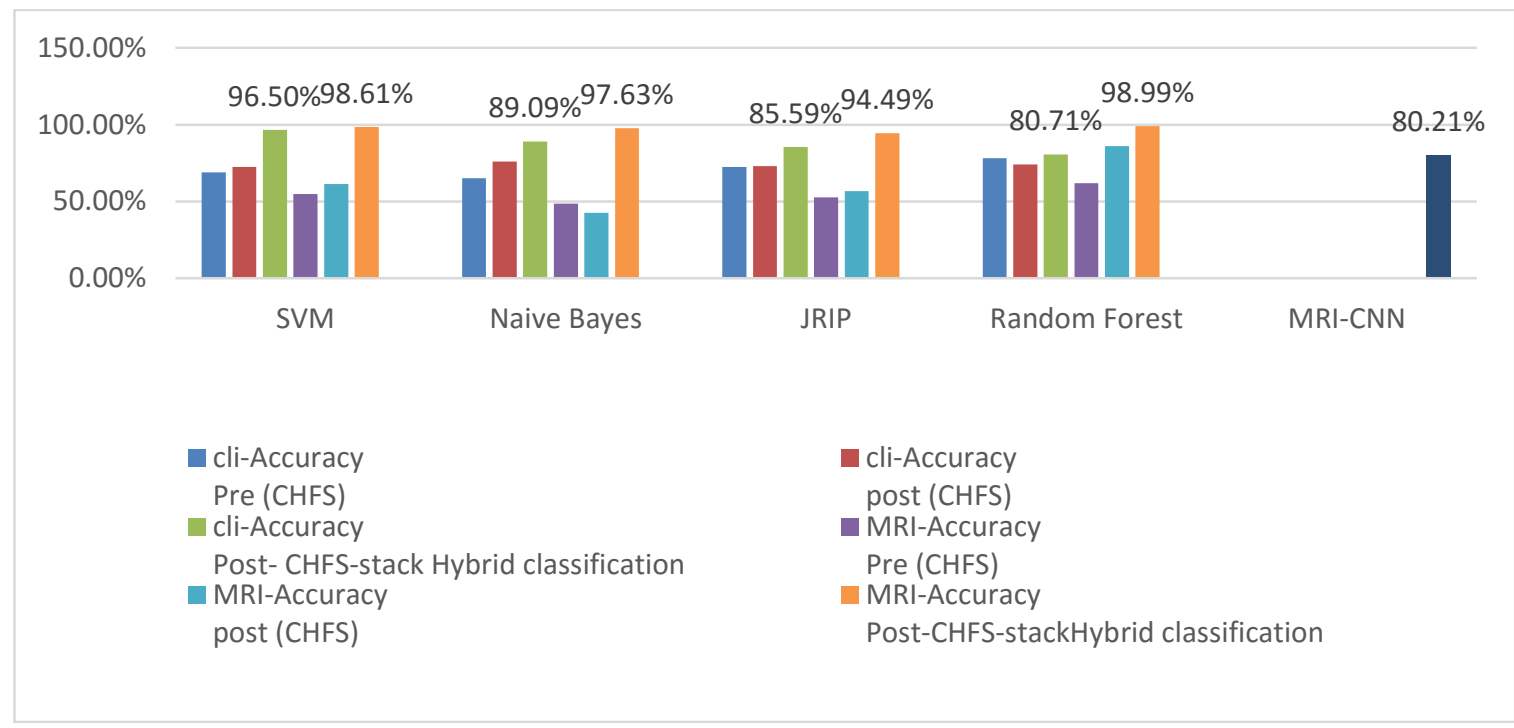

\section{Conclusion}

In this work, the author aims to early diagnosis of AD by using a benchmark Oasis Clinical dataset on our proposed composed hybrid feature selection (CHFS) model. This combines the advantages of three filter feature selection approaches and optimizes the Genetic Algorithm (OGA) by improving the initial population generating and genetic operators. Using the results of filter approaches as some prior information with using the $\mathrm{J} 48$ decision tree classifier as a fitness function instead of probability and random selection to speed up convergence and select the best features, After that using the selected feature in stack hybrid classification and combine three classifiers with improving the prediction and accuracy. The proposed model shows better than the traditional classification approaches for optimum feature selection and improvement of the classification process and effectively reduced the false-negative rate with high accuracy when using a support vector machine (SVM) as a meta-classifier in a hybrid classification method with $96.50 \%$ compared to $68.83 \%$ of usage individually which is considerably better than the previous state-of-the-art result. The results of the proposed model show an accurate classify Alzheimer's clinical samples at a low cost.Besides, Applying our proposed model on MRI imaging dataset, which contains 4 categories of AD patients. We aim to improve the MRI classification after our experiment on MRI dataset with CNN. This experiment result gives an $80.21 \%$ of accuracy classification. Also, The authors built a novel architecture feature extraction for the MRI dataset. Depending on the usage of four image filters to embedding the MRI images to numeric values that lead to the allowance of applying our CHFS model to select the best feature from MRI images. After that, move to the Stack hybrid classification process, which combines two classifiers of jrip and naïve Bayes with naïve Bayes as meta-classifier to get high accuracy of $98.99 \%$, and this result is better than CNN-MRI model of $80.21 \%$. Which consider better than the last state of the art result in neuroimaging classification, and our proposed show an accurate classify Alzheimer's disease. 


\section{References}

[1]Kalló, Gergö, Miklós Emri, et al. "Changes in the Chemical Barrier Composition of Tears in Alzheimer's Disease Reveal Potential Tear Diagnostic Biomarkers.” PLoS One, vol. 11, no. 6, Public Library of Science, June 2016, p. e0158000.

[2] J. Escudero, et al. Machine learning-based method for personalized and cost-effective detection of Alzheimer's disease. IEEE transactions on biomedical engineering 2013; 60(1): 164-168.

[3] Jyoti Soni et al. "Predictive Data Mining for medical diagnosis: An Overview of Heart Disease Prediction"International Journal of Computer Applications (0975 -8887), Volume 17, No. 8, March 2011."

[4] Williams, Jennifer A., Alyssa Weakley, Diane J. Cook, and Maureen SchmitterEdgecombe. (2013) "ML techniques for diagnostic differentiation of mild cognitive impairment." In Workshops at the Twenty-Seventh AAAI Conference on Artificial Intelligence: $71-76 \ldots$

[5] Chi, C. L., Oh, W., \&Borson, S. (2015) "Feasibility Study of a Machine Learning Approach to Predict Dementia Progression," In Healthcare Informatics (ICHI), International Conference: $450-450$

[6] Chyzhyk, D., \&Savio, A. (2010) "Feature extraction from structural MRI: data from OASIS database," University of The Basque Country, Internal research publication.

[7] S. R Bhagya Shree and Dr. H. S. Sheshadri "An initial investigation in the diagnosis of Alzheimer's disease using various classification techniques" IEEE ICCIC, 2014.

[8] Tina R. Patil and Mrs. S. S. Sherekar "Performance Analysis of Naive Bayes and J48 Classification Algorithm for Data Classification" International Journal of Computer Science and Applications Vol. 6, No.2, Apr 2013

[9] Jihad Ali et al. "Random Forests and Decision Trees" IJCSI International Journal of Computer IS, Vo. 9, Issue 5, No 3, September 2012

[10] Vemuri, P., Gunter, J. L., Senjem, M. L., Whitwell, J. L., Kantarci, K., Knopman, D. S., ... \& Jack, C. R. (2008) "Alzheimer's disease diagnosis individual subjects using structural MR images: validation studies," Neuroimage, 39(3): 1186-1197.

[11] Datta, P., and Shankle, W. R., \&Pazzani, M. (1996) “Applying ML to an Alzheimer's database," In Conference proceedings of the AAAI symposium: 25-27.

[12] Klöppel, S., Stonnington, C. M., Chu, C., Draganski, B., Scahill, R. I., Rohrer, J. D., ... \&Frackowiak, R. S (2008)“Automatic classification of MR scans in Alzheimer's disease." Brain, 131(3): 681-689.

[13] So, A., Hooshyar, D., Park, K. W., \& Lim, H. S. (2008) "Early Diagnosis of Dementia from Clinical Data by Machine Learning Techniques,” Applied Sciences, 7(7): 651.

[14] Ramirez, J., Górriz, J. M., Salas-Gonzalez, D., Romero, A., López, M., Álvarez, I., \& Gómez-Río, M. (2013) "Computer-aided diagnosis of Alzheimer's type dementia combining support vector machines and discriminant set of features." Information Sciences, 237: 59-72.

[15] Sheshadri, H. S., Shree, S. B., \& Krishna, M. (2015) "Diagnosis of Alzheimer's Disease Employing Neuropsychological and Classification Techniques," In IT Convergence and Security (ICITCS), 2015 5th International Conference: 1-6.

[16]Alzehimer's clinical medical dataset available online at https://www.oasisbrains.org/\#Data (accessed on 2 Jan 2019) OASIS-3: Principal Investigators: T. Benzinger, D. Marcus, J. Morris; NIH P50AG00561, P30NS09857781, P01AG026276, P01AG003991, R01AG043434, UL1TR000448, R01EB009352. AV-45 doses provided by Avid 
Radiopharmaceuticals, a wholly-owned subsidiary of Eli Lilly OASIS-3: https://doi.org/10.1101/2019.12.13.19014902

[17] Data mining: Practical machine learning tools and techniques by Ian H Witten and Eibe Frank, published by Elsevier, second edition 2008.

[18]Aouatif Amine Ali Elakad Mohammed Rziza Driss "GA-SVM and Mutual Information based Frequency Feature Selection for Face Recognition" GSCM-LRIT, Faculty of Sciences, Mohammed V University, B.P. 1014 Rabat, Morocco(2011).

[19] J.R. Quinlan, Induction of Decision Trees, Machine Learning 1: pp.81-106, Kluwer Academic Publishers, Boston, (1986).

[20] Leo Breiman. "Stacked Regressions" Machine Learning, 24, 49-64 (1996)

[21] Duarte Ferreira et al. "Applying data mining techniques to improve diagnosis in neonatal jaundice" BMC Medical Informatics and Decision Making, 0-5, 2012

[22] Jiawei Han, Micheline Kamber, Jian Pei "Data Mining: Concepts and Techniques" published by Elsevier, Third edition, 2012

[23] Anil Rajput, Ramesh Prasad Agarwal, Meghna Dubey S.P. Saxena, and Manmohan Raghuvanshi" J48 and JRIP Rules for EGovernance Data" International Journal of Computer Science and Security (IJCSS), Volume (5): Issue (2), Pp 201, 2011.

[24]Guyon I, Elisseeff A An introduction to variable and feature selection J Mach Learn Res 2003; 3(Mar):1157-82.

[25] Feature selection and classification systems for chronic disease prediction: A review Divya Jain, Vijendra SinghThe NorthCap University, Gurugram 12,2017, India, [online ] http://creativecommons.org/licenses/by-nc-nd/4.0

[26] Huang, C., A hybrid stock selection model using genetic algorithms and support vector regression. Applied Soft Computing, 2012. 12(2): p.807-818

[27] K. Tejeswinee, S.G. Jacob. Binary classification of Cognitive Disorders: Investigation on the Effects of Protein Sequence Properties in Alzheimer's and Parkinson's disease. IAENGIMECS 2017: 166-170

[28] Joliffe IT. Principal component analysis, series: Springer series in statistics. 2. Springer; NY: 2002

[29]O. Elsayed, K. Mahar, M. Kholief and H. A. Khater, "Automatic detection of the pulmonary nodules from CT images," 2015 SAI Intelligent Systems Conference (IntelliSys), IEEE, London, pp.742-746, 2015, DOI: 10.1109/IntelliSys.2015.7361223.

[30]Yasser Fouad, Hatem Khater, Mostafa Setta, Ashraf Alsaid, "proposed Approach for Automatic Underwater Object Classification," International Journal of Research and Surveys, ICIC -EL, Volume 12, No. 12, pp. 1202-1212, 2018, DOI:10.24507/icicel.12.12.1205.

[31] The national center for health statistics report for 2019 (https://www.cdc.gov/nchs/fastats/leading-causes-of-death.htm).

[32] Weka open source software for data mining availble online (https://www.cs.wakito.ac.nz/) accessed 26 Dec 2019.

[33] Chen, F. and F. Li, A combination of feature selection approach with SVM in credit scoring. Expert Systems with Applications, 2010. 37(7): p. 4902-4909.

[34] Chen, Y. -W., and Lin, C. -J., Combining SVMs with various feature selection Strategies, Available from http://www.csie.ntu.edu.tw/ cjlin/papers/features.pdf, 2005.

[35] Dash, M., and H. Liu, Feature Selection for Classification. Intelligent Data Analysis, 1997: p. 131C156. 
[36] Devi, K.N., V.M. Bhaskaran and G.P. Kumar, Cuckoo ,Optimized SVM for Stock Market Prediction, IEEE Sponsored 2nd International Conference on Innovations in Information, Embedded, and Communication Systems (ICJJECS)2015, 2015.

[37] Dai, W., Y.E. Shao, and C. Lu, Incorporating feature selection method into support vector regression for stock index forecasting. Neural Computing and Applications, 2013. 23(6): p. 1551-1561.

[38] Yanan Mao, Zuoquan Zhang, Dingyuan Fan, "Hybrid feature selection based on improved genetic algorithm for stock prediction," 2016 6th International Conference on Digital Home, 2016.

[39] Dina A. Ragab, Maha Sharks and Omneya Attallah," Breast Cancer Diagnosis Using an Efficient CAD System Based on Multiple Classifiers," Diagnostics 2019, 9, 165; DOI:10.3390/diagnostics9040165.

[40] David P Salmon and Mark W. Bondi“Neuropsychological Assessment of Dementia" Access NIH public, PubMed central, US national library of medicine Institutes of Health, May 2010.

[41] Divya Jain, Vijendra Singh," Feature selection and classification systems for chronic disease prediction: A review," Egyptian Informatics Journal 19 (2018) 179-189,2018

[42] Ramon Casanova, Fang-Chi Hsu, Kaycee M Sink, Stephen R Rapp, Je_ D Williamson, Susan M Resnick, Mark A Espeland, Alzheimer's Disease Neuroimaging Initiative, et al. Alzheimer's disease risk assessment using large-scale machine learning methods. PloS one, 8(11):e77949, 2013.

[43] Susanne G Mueller, Michael W Weiner, Leon J Thal, Ronald C Petersen, Cli_ord Jack, William Jagust, John Q Trojanowski, Arthur W Toga, and Laurel Beckett. The Alzheimer's disease neuroimaging initiative. Neuroimaging Clinics of America, 15(4):869\{877, 2005.

[44] Elizabeth G Kehoe, Jonathan P McNulty, Paul G Mullins, and Arun LW Bode.Advances in MRI Biomarkers for the Diagnose of AD. Biomarkers in Medicine, 8(9):1151\{1169, 2014.

[45] Arthur W Toga and Karen L Crawford. The AD Neuroimaging informatics core: A decade in review. Alzheimer's and Dementia: The Journal of the Alzheimer's Association, 11(7):832\{839, 2015.

[46] Michael W Weiner, Dallas P Veitch, Paul S Aisen, Laurel A Beckett, Nigel J Cairns, Jesse Cedarbaum, Robert C Green, Danielle Harvey, Cli_ord R Jack, William Jagust, et al. 2014 Update of the AD Neuroimaging: A review of papers published since its inception. Alzheimer's and Dementia: The Journal of the Alzheimer's Association, 11(6):e1\{e120, 2015. [47]A benchmark Alzheimer's MRI segmentation Dataset from online https://www.kaggle.com/tourist55/alzheimers-dataset-4-class-of-images (accessed online 12/05/2019).

[48]N. Woods, O. Longe, and A. Roberts, "A Sobel edge detection algorithm based system for analyzing and classifying image-based spam," Journal of Emerging Trends in Computing and Information Sciences, vol. 3, no. 4, 2012.View at: Google Scholar.

[49] G.Saianilkumar1, K.Ravindrakbabu2, B.V. Nagendra Prasad Reddy3, "Medical Image Retrieval Based On Edge Histogram Descriptor," International Journal of Engineering Science Invention ISSN (Online): 2319 - 6734, ISSN (Print): 2319 - 6726 www.ijesi.org |Volume 4 Issue 4 || April 2015 || PP.22-27.

[50] Jianfang Cao," Implementing a Parallel Image Edge Detection Algorithm Based on the Otsu-Canny Operator on the Hadoop Platform, "Computational Intelligence and Neuroscience, Volume 2018 |Article

ID 3598284 | 12 pages | https://doi.org/10.1155/2018/3598284 